\title{
Über den Einfluß \\ von Substitution in den Komponenten binärer Lösungsgleichgewichte
}

XI. Mitteilung

Die binären Lösungsgleichgewichte zwischen Phenol, beziehungsweise den drei isomeren Nitrophenolen einerseits, den drei isomeren Phenylendiaminen andererseits

\author{
Von \\ Robert Kremann und Bruno Petritschek \\ Aus dem Chemischen Institut der k. k. Universität in Graz \\ (Mit 12 Textfiguren)
}

(Vorgelegt in der Sitzung am 8. Juni 1917)

Die Lösungsgleichgewichte der zwölf binären Sysieme, wie sie sich aus den im Titel genannten Stoffen aufbauen lassen, stellen ziemlich die interessantesten der bisher untersuchten Systeme dieser Mitteilungsfolge dar. Während in letzteren im Falle des Vorliegens einer Verbindung im festen Zustande fast immer nur eine Verbindung der beiden Komponenten des binären Systems als neuer Bodenkörper auftritt und diese Verbindung in der Regel sich aus je einem Molekül der beiden Komponenten zusammensetzt, beobachtet man in den in vorliegender Mitteilung behandelten Systemen die allergrößte Mannigfaltigkeit sowohl in bezug auf die Zahl als auch die Zusammensetzung der neu auftretenden Bodenkörper. Diese Beobachtungen möchten wir daher als wertvolle Bausteine für die Valenzauffassung im Sinne der Darlegungen in früheren Mitteilungen auffassen. Der Vorgang der Bildung von 
Verbindungen zweier Komponenten läßt sich nach zwei Seiten hin charakterisieren:

1. In bezug auf die Anzahl der Verbindungen, welche $z w e i$ Teilstoffe je nach ihrem Verhältnis im Schmelzfluß im festen Zustande bilden;

2. in bezug auf das molekulare Verhältnis, in dem beide Teilstoffe in ibren Verbindungen vertreten sind.

Das molekulare Verhältnis der Teilstoffe in ihren Verbindungen wird bestimmt durch den Wert der Restvalenzen der beiden Teilstoffe, d. h. dem Verhältnis der Anzahl gelockerter Valenzelektronen, die an den einzelnen Teilstoffen vorhanden sind, beziehungsweise der durch diese bedingten Kraftfeldzentren. Bei annähernd gleicher Anzahl solcher Kraftfeldzentren auf beiden Teilstoffen wird in der flüssigen Mischung jeweils die äquimolekulare Verbindung mit ihrer Konzentration vorherrschen, also im festen Zustande zur Abscheidung gelangen. Ist hingegen die Restvalenz der einen Komponente, also die Anzahl der Valenzkraftfelder auf ihr wesentlich größer, als es in bezug auf die zweite Komponente der Fall ist, so wird sich in den flüssigen Mischungen eine an Molekülen der zweiten Komponente reichere Verbindung in maximaler Konzentration bilden, also zur Abscheidung im festen Zustande kommen.

Diese Erwägungen gelten alle unter der Voraussetzung genügend großer totaler Affinität der Komponenten zueinander. Wir dürfen uns nämlich nicht vorstellen, daß in der flüssigen Schmelze restlos sich ein Gleichgewicht zwischen der betreffenden im festen Zustande isolierten Verbindung und ihren Komponenten einstellt, die Verbindung also direkt in ihre Komponenten zerfällt. Ganz im Gegenteil werden allgemein diese Verbindungen je nach der Zusammensetzung der Schmelze über andere Verbindungen, die an der ersten oder der zweiten Komponente reicher sind, je nachdem die erste oder die zweite Komponente im Schmelzfluß überwiegt, in die reinen Komponenten zerfallen.

$\mathrm{Ob}$ nun in einem binären System nur eine oder mehrere dieser Verbindungen im festen Zustande zur Abscheidung kommen, ist eine Frage des Konzentrationsbetrages der ein- 
zelnen Verbindungen und dieser ist letzten Endes eine Frage der Affinität der beiden Komponenten. Während wir unter Restvalenz, wie Valenz ïberhaupt, eine Qualitätsgröße zu verstehen haben, hat die totale Affinität die Bedeutung einer Quantitätsgröße: der Intensität der gegenseitigen Kraftlinien eines elektromagnetischen Kraftfeldes, hervorgerufen durch die Betätigung der gelockerten Valenzelektronen, die die einzelnen Moleküle der T eilkomponenten ausstrahlen. Ist die Intensität derselben für ein bestimmtes Verhältnis der beiden Teilstoffe, das eben durch das Verhältnis der Anzahl der Valenzkraftfelder derselben bestimmt ist, eine genügend große, so wird in den Schmelzen beider Stoffe der allerverschiedensten Zusammensetzung stets die Konzentration der Verbindung dieser Zusammensetzung in hohem Maße überwiegen gegenüber den Konzentrationen der Verbindungen der übrigen $\mathrm{Zu}$ sammensetzungen, weil eben die Moleküle des im Überschuß vorhandenen Teilstoffes mehr oder minder vollkommen unter Bildung von Molekülen der bestimmten Verbindung gebunden werden. Es verbleiben also nur wenig freie Moleküle dieser Komponente, die mit den Molekülen der im Überschuß vorhandenen Komponente zu Verbindungen anderer Zusammensetzung zusammentreten können. Da die Konzentration dieser letztgenannten Verbind ungen im Verhältnis zu der der erstgenannten Verbindung $n$ ur klein ist, wird sich nur diese (neben den reinen Kom ponenten, wenn diese in entsprechend starkem Überschuß vorhanden sind) im festen Zustande abscheiden.

Mit sinkender Intensität der einzelnen Valenzkrdftfelder mit steigender Zahl derselben auf einem Molekül dürfte die Intensität der einzelnen Valenzkraftfelder für sich betrachtet abnehmen - verbleibt in der Schmelze von der im Unterschuß vorhandenen Komponente ein steigender Anteil dieser Komponente zunächst ungebunden durch die im Überschuß vorhandene Komponente nach Art der bestimmt zusammengesetzt angenommenen Verbindung. Es kann sich hiermit jeweils eine an der übe rschüssigen Komponente reichere Verbindung mit höheren Konzentrationsbeträgen bilden, so daß also je nach der Konzentration der Schmelze infolge der 
Massenwirkung die an der einen oder anderen Komponenie reichere Verbindung mit ihrer Konzentration superponieren und zur Abscheidung kommen kann. In diesen Fällen wird also die Zusammensetzung der jeweils abgeschiedenen Verbindung eine Funktion der Zusammensetzung der Schmelze. Wir dürfen also im Sinne dieser Ausführungen sagen: Ist die in obigem Sinne definierte totale Affinität groß, was im allgemeinen bei erheblich großer Heteropolarität der Komponenten der Fall sein wird, wird man im Zustandsdiagramm in der Regel nur das Auftreten einer Verbindung bestimmter Zusammensetzung beobachten. Bei Verminderung der gegenseitigen totalen Affinität, die im Sinne der Darlegungen in den früheren Mitteilungen auch durch räumliche Behinderung bedingt sein kann, ${ }^{1}$ wird die Möglichkeit des Auftretens mehrerer Verbindungen gegeben. sein, die letzten Endes ganz verschwinden können, wenn die Konzentration dieser Verbindungen in der Schmelze genügend weit unterhalb der Konzentration der Teilkomponenten bleibt.

Im Sinne dieser Darlegungen haben wir nun die Versuchsergebnisse bei der Aufnahme der zwölf Zustandsdiagramme von Phenol, beziehungsweise den drei isomeren Nitrophenolen einerseits, den drei isomeren Phenylendiaminen andererseits aufgefaßt. Von den Systemen Phenol einerseits, den drei isomeren Phenylendiaminen andererseits liegen die Verhältnisse beim System Phenol-p-Phenylendiamin am einfachsten.

Aus dem in Fig. 1 dargestellten Zustandsdiagramm geht die ausschließliche Existenz ein er Verbindung der Zusammensetzung von 2 Molekülen Phenol und 1 Molekül $p$-Phenylendiamin hervor.

Die Existenz einer Verbindung dieser Zusammensetzung läBt sich schon nach dem einfachen ganzzahligen Valenz-

1 Möglicherweise führt sich diese räumliche Valenzbehinderung zurück auf die Tatsache, daß zwei auf einem Molekül befindliche Kraftfelder sich gegenseitig schwächen und dies um so mehr, je näher sie bei einander liegen. So würde sich, wenn man bei gewissen Disubstitutionsprodukten des Benzols an den Substituenten die Kraftfeldzentren annimmt, die cet. par. geringste Totalaffinität bei den $o$-Substitutionsprodukten gegenüber den $m$ - und $p$-Substitutionsprodukten erklären. 
schema übersehen. Phenole geben mit Monaminen in der Regel äquimolekulare Verbindungen. ${ }^{1}$ Wenn wir im Phenol ein Valenzkraftfeld, in den Amiden den Sitz des Valenzkraftfeldes am Stickstoff annehmen, so ist es verständlich und

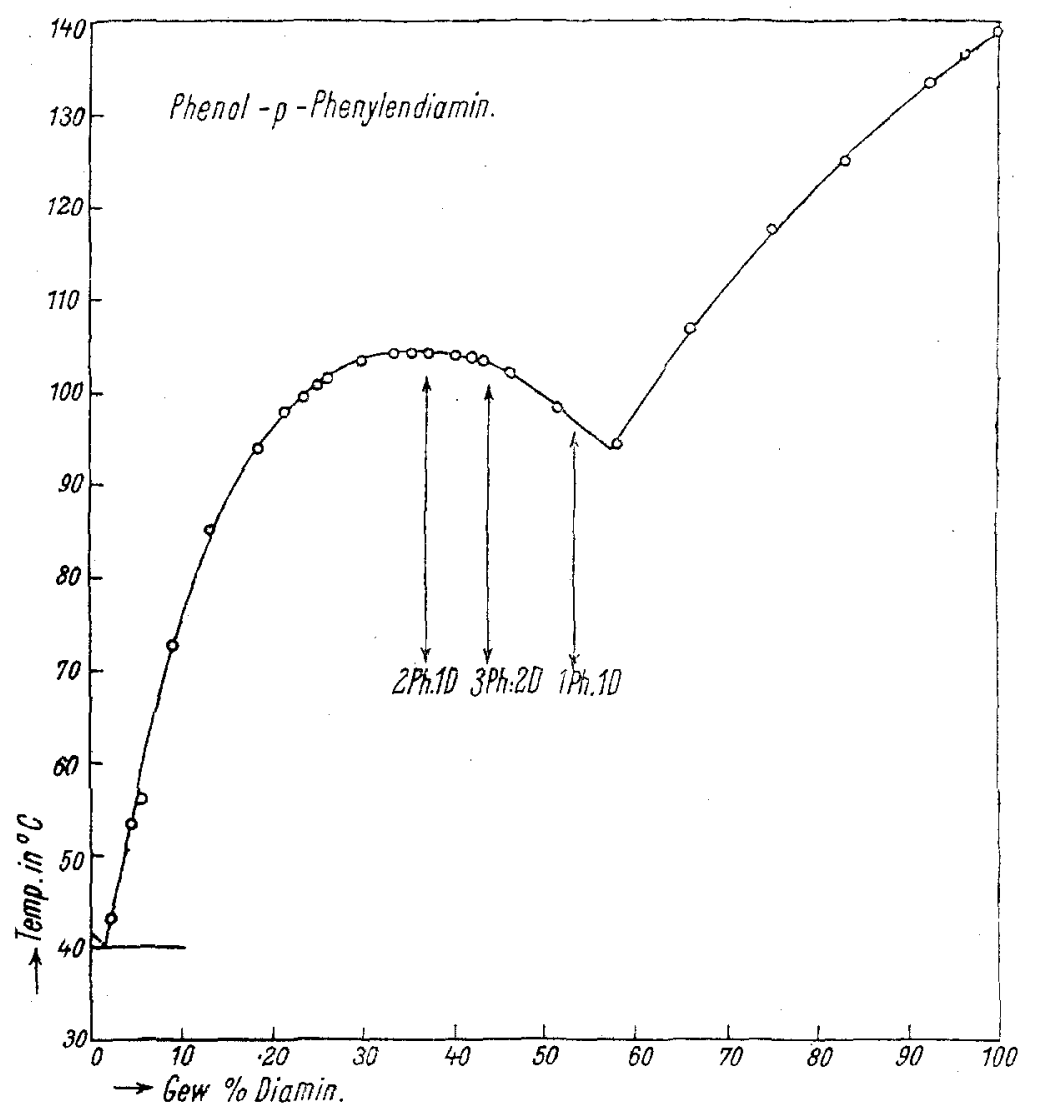

Fig. 1.

naturgemäß, daß 1 Molekül Phenylendiamin 2 Moleküle Phenol $z \mathrm{u}$ binden vermag, ebenso wie z. B. Harnstoff ${ }^{2}$ und symmetrischer Dimethylharnstoff ${ }^{3}$ je 2 Moleküle Phenol zu binden

1 Kremann, Monatsh. f. Chemie, 27, 91 (1906); cf. Philip, Journ. Chem. Soc., 83, 828 (1903).

2 R. Kremann, ibid., 28, 1125 (1907).

3 R. Kremann; ibid., 31, 843 (1910). 
vermögen. Auf Grund früherer Untersuchungen hat sich ergeben, daß von den drei isomeren Benzoldisubstitutionsprodukten die Neigung zur Bildung von Verbindungen, also die Totalaffinität gegen eine zweite Komponente in der Reihe $p, m, o$ abnimmt. Die Verminderung der totalen Affinität beim $m$-, beziehungsweise o-Phenylendiamin gegenüber Phenol kann, wenn sie in der Reihe der Phenylendiamine überhaupt zum Ausdruck kommt, sich in zweierlei Weise bemerkbar machen. Entweder es nimmt im Sinne obiger Ausführungen die Zahl der im

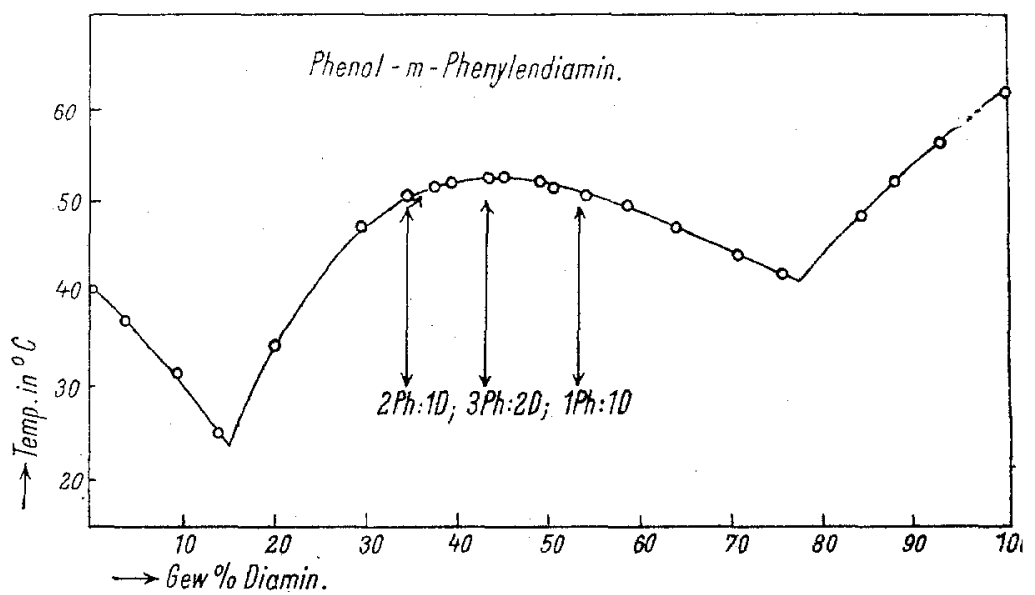

Fig. 2.

festen Zustande abscheidbaren Verbindungen zu, beziehungsweise es kommt überhaupt nicht mehr zur Abscheidung von Verbindungen im festen Zustande. Es kann sich aber auch die Verminderung der Totalaffinität, $\mathrm{d}$. b. die Verminderung der Summe der Intensitäten der beiden Kraftfelder des Phenylendiamins durch Näherung der beiden Amidogruppen in der $m$-, beziehungsweise $o$-Verbindung dahin äußern, daß die Totalaffinität zwar noch groß genug ist, daß im gesamten Konzentrationsgebiet außer den Komponenten sich gleichfalls zwar nur eine Verbindung abscheidet, aber zu klein, daß es zur Bindung einer Verbindung von 2 Molekülen Phenol durch 1 Molęuül Diamin kommt, sondern zur Abscheidung einer phenolärmeren Verbindung. In der Tat haben wir denn auch, wie das Zustands- 
diagramm in Fig. 2 es zeigt, im System Phenol-m-Phenylendiamin einé Verbindung vom homogenen maximalen Schmelzpunkt von $52^{\circ}$ der Zusammensetzung von 2 Molekülen Diamin und 3 Molekülen Phenol als der alleinig vorliegenden feststellen können. Wir dürfen uns also vorstellen, daß die Intensität der beiden. Valenzkraftfelder des $m$-Phenylendiamins nicht mehr ausreicht, um, wie es beim $p$-Phenylendiamin der

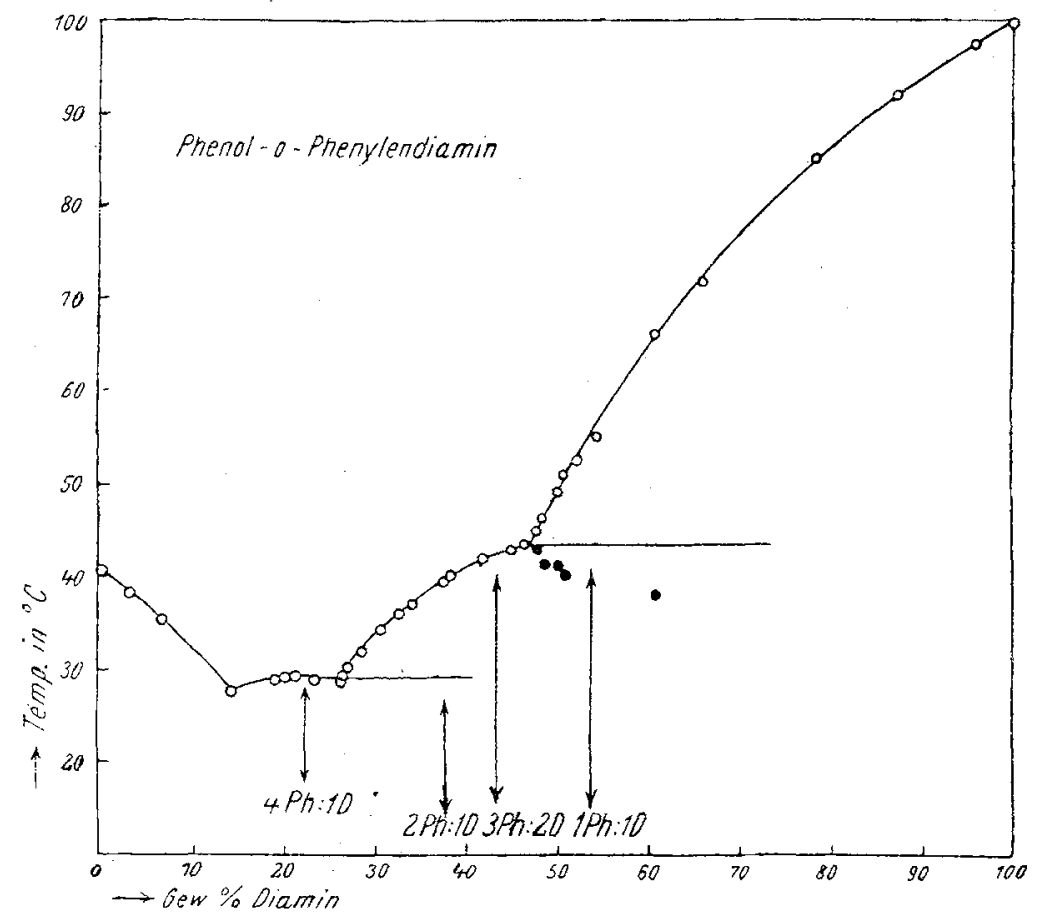

Fig. 3.

Fall ist, 2 Moleküle Phenol in solchen Konzentrationsbeträgen zu binden, daß es zur Abscheidung dieser Verbindung im festen Zustande kommt. Das $m$-Phenylendiamin vermag nur mehr $11 / 2$ Moleküle, d. h. also 2 Moleküle $m$-Phenylendiamin, demnach 3 Moleküle Phenol $z u$ binden. Diese Verbindung bildet sich aber mit so erheblichen Konzentrationsbeträgen, daß sie neben den reinen Komponenten als alleinige Verbindung sich abscheidet. Im System o-Phenylendiamin - Phenol 
sehen wir im Sinne obiger Ausführungen eine weitere Verminderung der totalen Affinität, indem hier, wie Fig. 3 es zeigt, zwei Verbindungen eine phenolreichere und eine phenolärmere vorliegen. Aus phenolreichen Mischungen und bei tieferen Temperaturen scheidet sich eine Verbindung von 4 Molekülen Phenol und 1 Molekül o-Phenylendiamin ab, während die nächst diaminreichere Verbindung jedenfalls die äquimolekulare Verbindung ist. Beide Verbindungen scheinen, wie der Grad der Abflachung der ihnen zugehörigen Teile der Schmelzkurve zeigt, stark im Schmelzfluß dissoziiert.

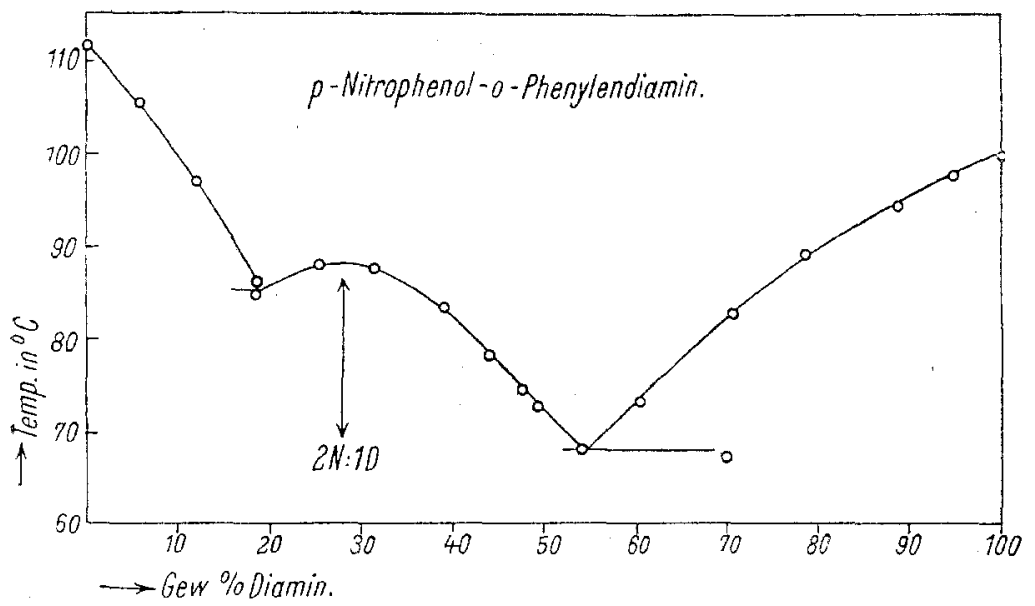

Fig. 4.

Im Sinne obiger Ausführungen sehen wir also, daß auch bei den drei isomeren Phenylendiaminen die totale Affinität gegenüber Phenol in der Reihe $p, m, o$ abnehmen dürfte, wenngleich die Abnahme hier graduell nicht so stark sein dürfte wie in anderen Fällen, wo cet. par. nur das $p$ - und $m$-Isomere Verbindungen liefert, im System mit den o-Isomeren es aber zur Abscheidung von Verbindungen nicht mehr kommt. ${ }^{1}$

Wenn wir die neun binären Systeme der drei isomeren Diamine mit den drei isomeren Nitrophenolen betrachten, so sehen wir, daß hier weniger das Diamin den Diagrammhabitus,

1 Siehe den zusammenfassenden Bericht in der VIl. Mitteilung von R. Kremann und G. Grasser, Monatsh. f. Ch., 37, 723 (1916). 
d. h. die Neigung zur Bildung von Verbindungen nach Art und Zahl bestimmt, als vielmehr das Nitrophenol und in bezug auf dieses auch wieder die totale Affinität in der Reihe $p$-, $m$-, o-Nitrophenol abnimmt.

So ergibt sich, daß $p$-Nitrophenol sowohl mit $o$ - als mit $m$-Phenylendiamin nur eine Verbindung bildet, die die normale Zusammensetzung: 2 Moleküle Nitrophenol und 1 MolekülDiamin

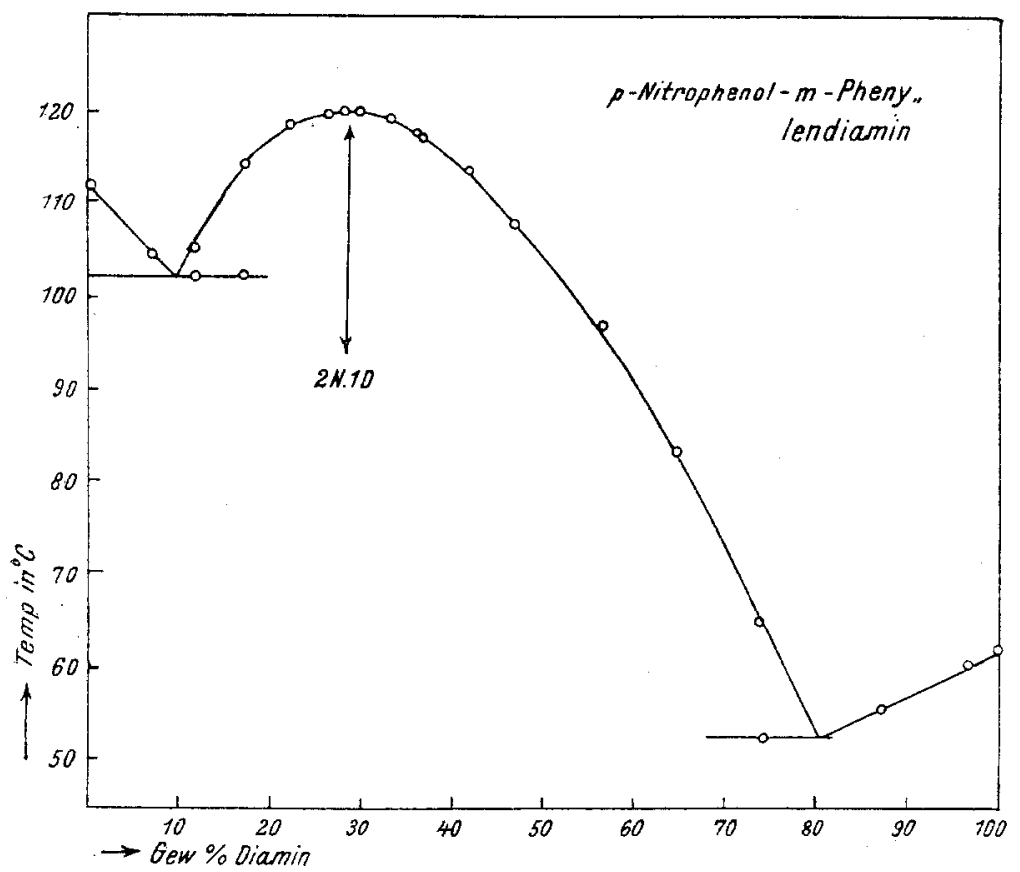

Fig. อ.

haben, wie aus den Figuren 4 und 5 hervorgeht. Nur das System $p$-Phenylendiamin- $p$-Nitrophenol zeigt insofern ein abweichendes Verhalten, indem, wie Fig. 6 es zeigt, hier zwei Verbindungen vorliegen, die der Zusammensetzung 4 Moleküle $p$-Nitrophenol und 1 Molekül $p$-Phenylendiamin, beziehungsweise 1 Molekül $p$-Nitrophenol und 1 Molekül $p$-Phenylendiamin entsprechen. Die Verbindungen haben die gleiche Zusammensetzung wie es der Fall ist im System Phenol-o-Phenylendiamin. 
Wenn wir die Verhältnisse in diesem System gegenüber den Systemen von Phenol und den beiden anderen Diaminen auf eine Verminderung der totalen Affinität zurückgeführt hatten, so haben wir für diese Verminderung die räumliche Anordnung in der Orthostellung verantwortlich gemacht. Beim System $p$-Diamin- $p$-Nitrophenol, wo beide Komponenten in der für die Verbindungsfähigkeit günstigsten Stellung, der $p$-Stellung, sich befinden, können wir die Abnahme der totalen Affinität der beiden Komponenten in diesem System gegenüber den Systemen $p$-Nitropheriol mit $m$-, beziehungsweise

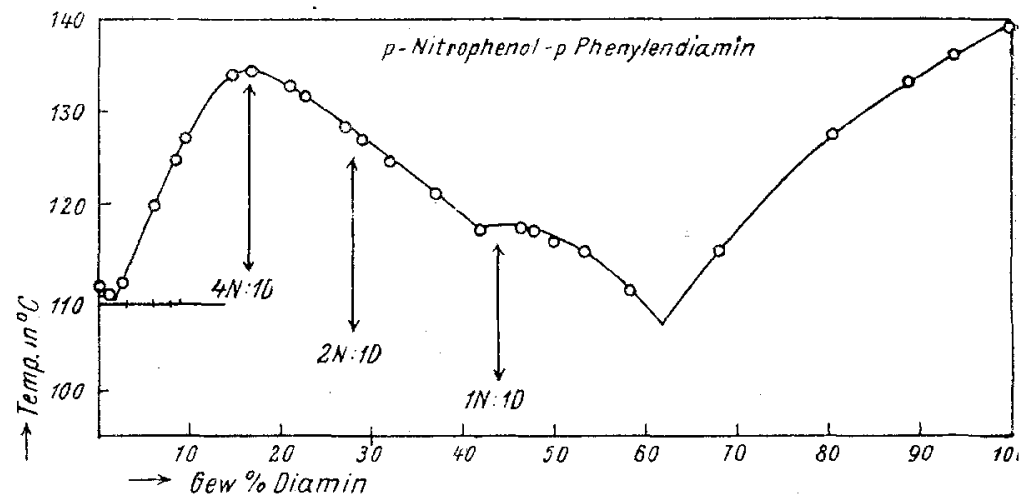

Fig. 6.

o-Diamin dadurch erklären, daß hier die Temperatur, bei der die Abscheidung der Verbindungen erfolgt, höher ist als in den beiden anderen Systemen, bei denen es nur zur Abscheidung einer Verbindung kommt, die Verminderung der totalen Affinität also hier auf die höhere Abscheidungstemperatur zurückzuführen ist. Ganz allgemein kann ja gesagt werden, daß bei der vergleichen den Betrachtung solcher binärer Zustandsdiagramme die Verschiedenheit der Temperatur, bei der wir eine solche vorzunehmen gezwungen sind, einen die allgemeinen Gesetzmäßigkeiten verwischenden Einfluß ausübt.

Die Verminderung der Affinität der Komponenten in der Reihe $p$-, $m-, o$-Nitrophenol wird am augenfälligsten, wenn wir die Systeme der drei Phenylendiamine mit o-Nitrophenol 
betrachtet. Wie aus den Figuren 7, 8 und $9 \mathrm{zu}$ sehen ist, gibt $o$-Nitrophenol mit keinem der drei isomeren DiamineVerbindungen im festen Zustande, sondern nur je ein einfaches Eutektikum. Das $m$-Nitrophenol steht in seinem Verhalten gegenüber den drei isomeren Phenylendiaminen

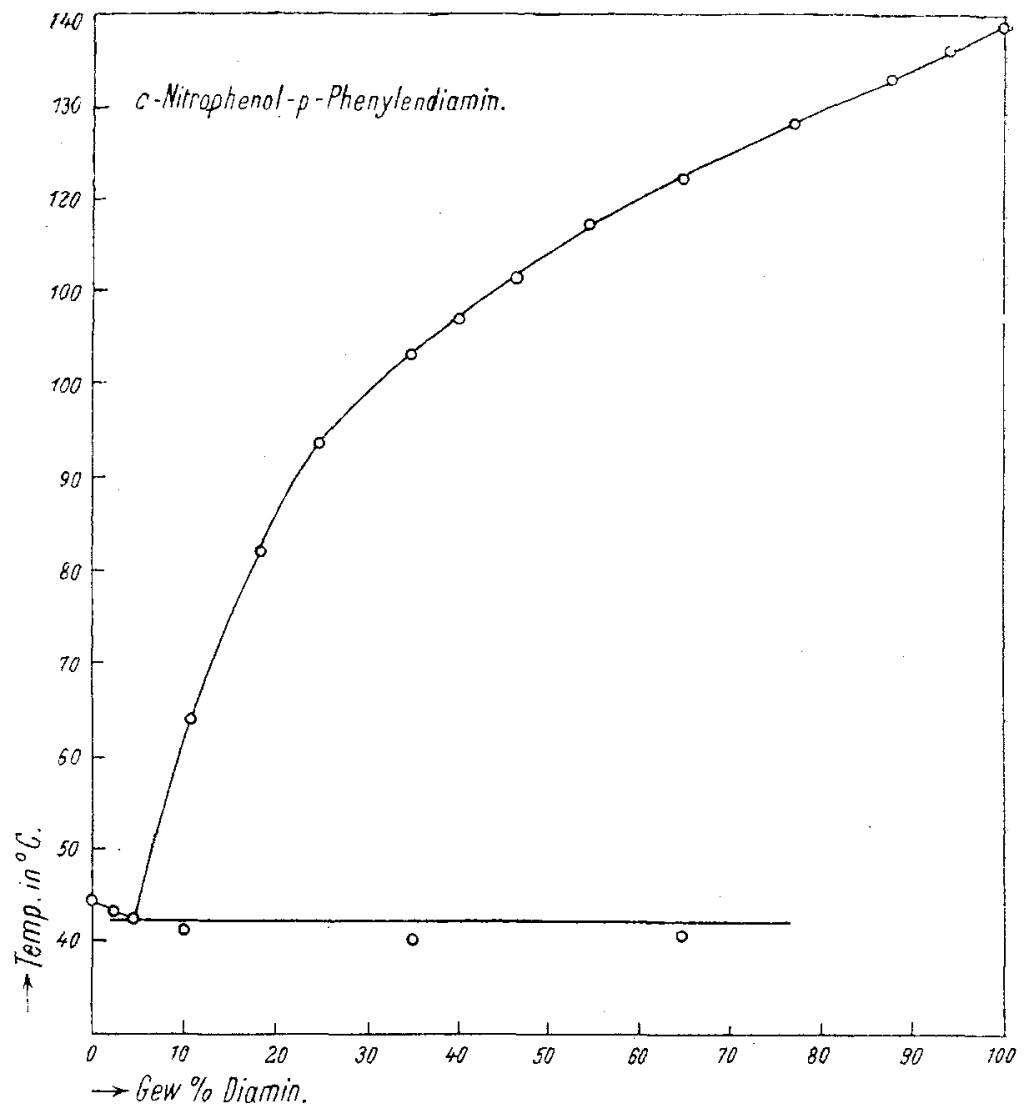

Fig. 7.

zwischen dem $o$ - und $p$-Nitrophenol, und zwar etwas näher dem letzteren. $m$-Nitrophenol liefert nämlich mit allen drei isomeren Phenylendiaminen mehr als je eine Verbindung, und zwar mit sämtlichen drei isomeren Diaminen, wie aus den Figuren 10 bis 12 hervorgeht, je eine Verbindung; bestehend aus 2 Molekülen $m$-Nitrophenol und 1 Molekül Diamin. In den Systemen $m$-Nitrophenol-.-o-Phenylendiamin, beziehungsweise 
$m$-Phenylendiamin liegt ferner je eine äquimolekulare Verbindung der beiden Komponenten, im System $m$-Nitro-

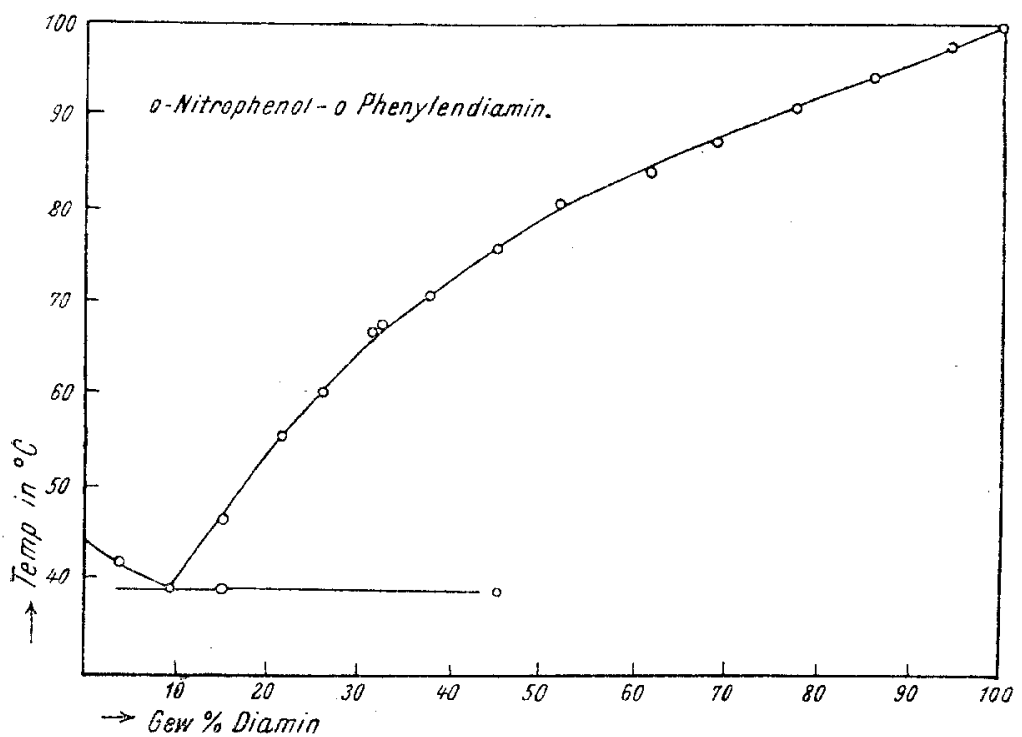

Fig. 8.

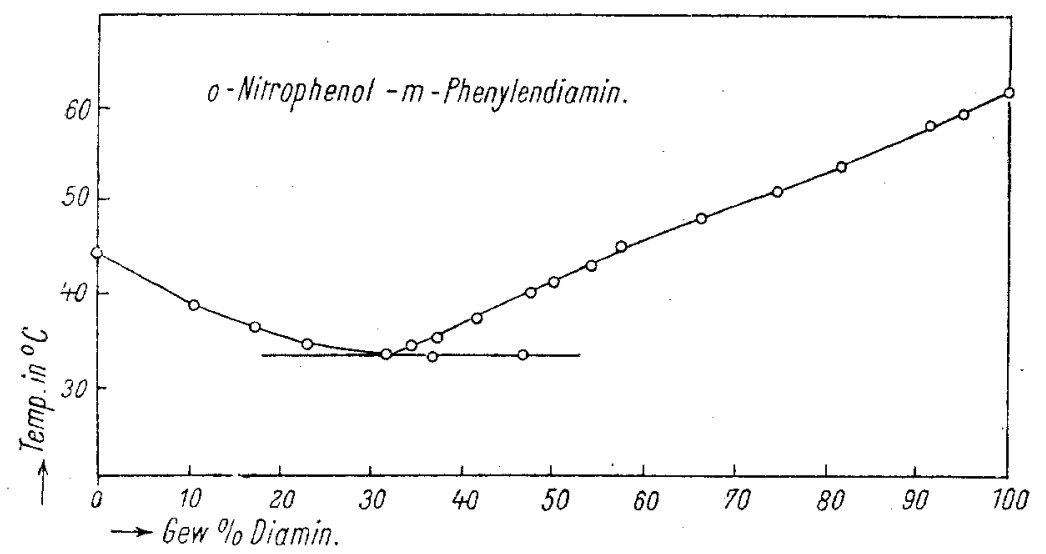

Fig. 9.

phenol $-p$-Phenylendiamin als zweite Verbindung eine solche, bestehend aus 1 Molekül Nitrophenol und 2 Molekülen Diamin, vor. Außerdem existiert noch im System $m$-Nitrophenol- 
$p$-Phenylendiamin eine dritte diaminreichere Verbindung der vermutlichen Zusammensetzung von 1 Molekül Nitrophenol und 4 Molekülen Diamin.

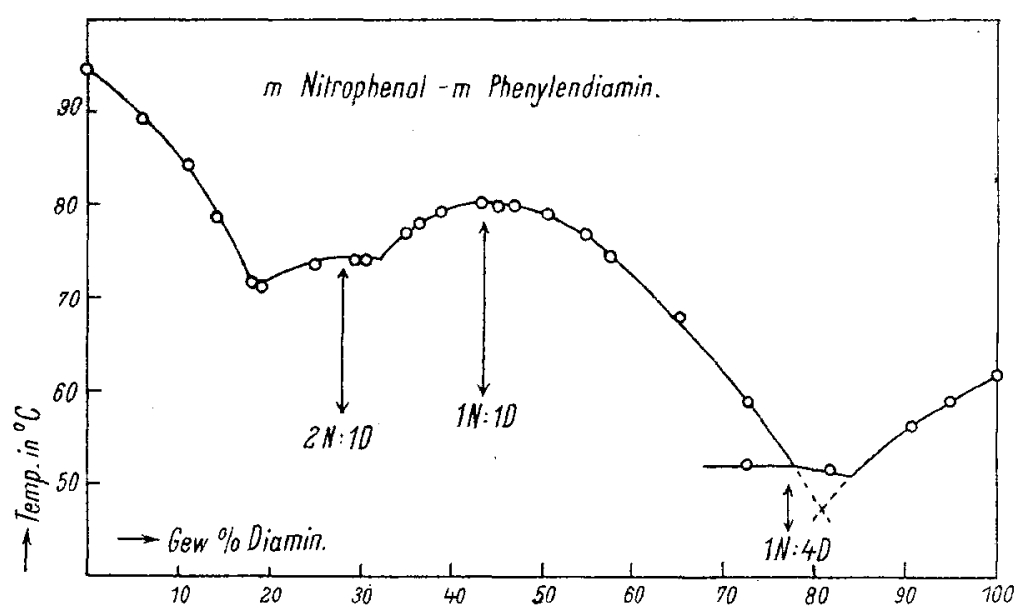

Fig. 10.

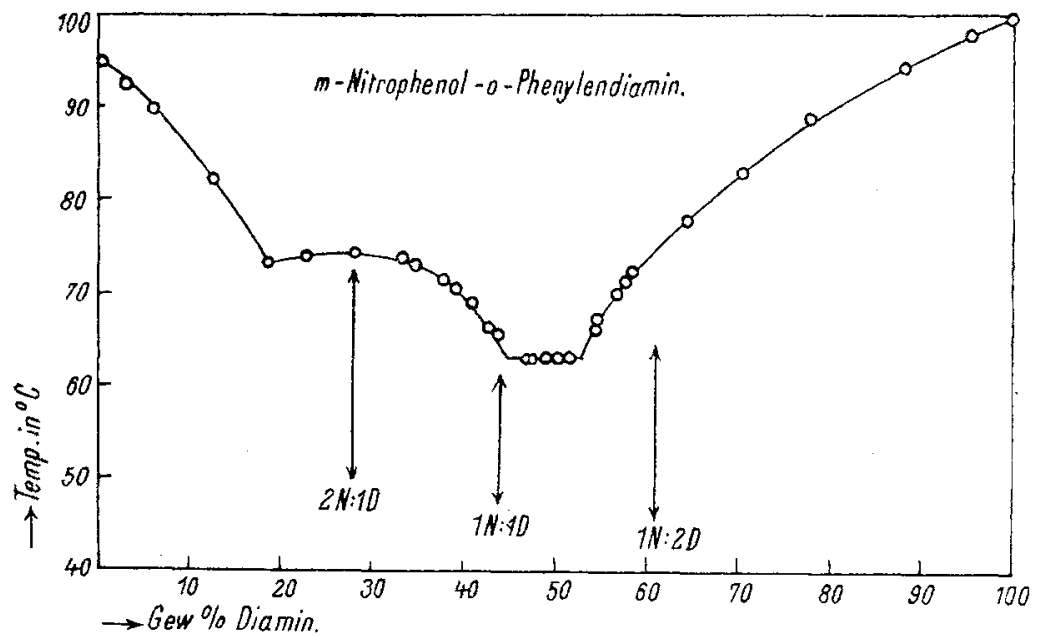

Fig. i 1.

Beim Vergleich des Einflusses der gegenseitigen Stellung der beiden Amidogruppen in den drei isomeren Phenylendiaminen auf die Verbindungsfähigkeit mit Phenol und den 
drei isomeren Nitrophenolen sieht man, daß im Sinne obiger Ausführungen auch bei den Phenylendiaminen in der 0 -Stellung der Amidogruppen eine sterische Valenzbehinderung angedeutet

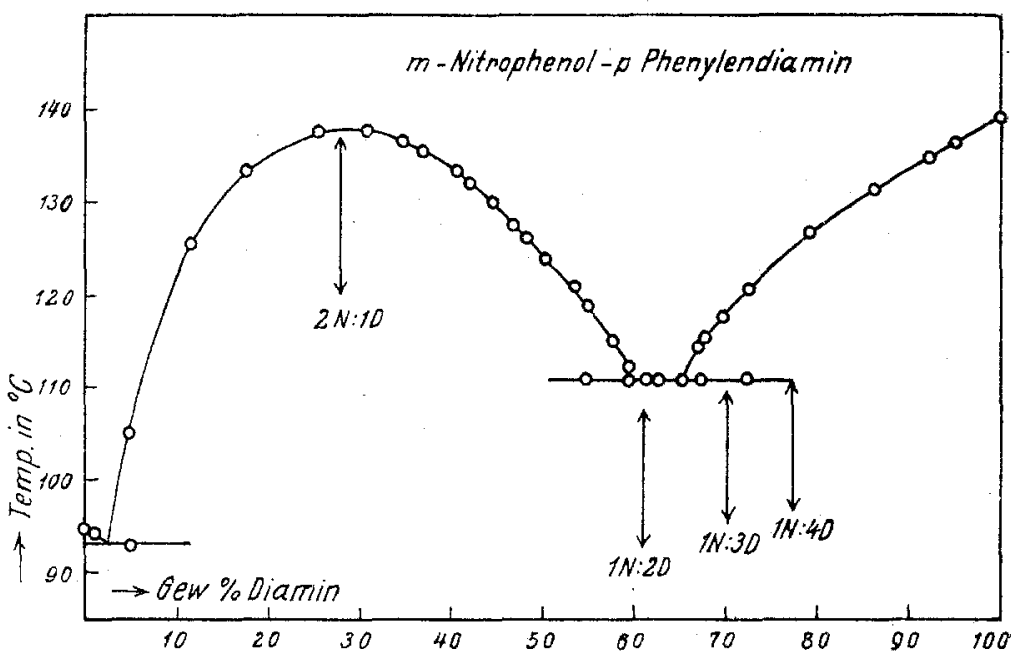

Fig. 12.

ist, wenngleich sie nicht so hervorstechend ausgeprägt ist, als dies bei der Substitution in der Orthostellung bei den Dinitrobenzolen, beziehungsweise den Nitrophenolen beobachtet wurde.

\section{Experimenteller Teil.}

Die Methode der Aufnahme der Zustandsdiagramme war die im besonderen in den letzten Mitteilungen genauer präzisierte, weshalb wir uns darauf beschränken, die tabellarische Wiedergabe der Versuchsresultate und ihre Diskussion folgen zu lassen.

1. Die Systeme Phenol und die drei isomeren Phenylendiamine.

Wie aus Tabelle 1 und Fig. 1 zu sehen ist, läuft der einen neuen Bodenkörper anzeigende Ast durch ein abgeflachtes Maximum bei $104.9^{\circ}$ bei 37 bis 36 Gewichtsprozenten Diamin. Da der Zusammensetzung einer Verbindung 
von 1 Molekül Diamin und 2 Molekülen Phenol ein Prozentgehalt von $36 \cdot 7 \%$ Diamin entspricht, liegt also in diesem System diese Verbindung als reiner Bodenkörper vor. Sie ist die einzige Verbindung beider Komponenten. Ihr Eutektikum mit Phenol liegt nahe bei reinem Phenol, d. i. bei $40^{\circ}$ und $1.5 \%$ Diamin, ihr Eutektikum mit $p$-Phenylendiamin bei $94^{\circ}$ und rund $58 \%$ Diamin. Im System Phenol $-m$-Phenylendiamin zeigt (Tabelle 2 und Fig. 2) der hier gleichfalls auftretende Ast eines neuen Bodenkörpers ein Maximum, das etwa 43 bis $44 \%$ Diamin entspricht und bei $52 \cdot 6^{\circ}$ liegt. Die Zusammensetzung der Verbindung ist demgemäß eine andere. Sowohl die Mischungen, denen eine Zusammensetzung von 1 Phenol und 1 Diamin entspricht, als auch die mit 2 Molekülen Phenol und 1 Molekül Diamin liegen bereits am ab-, beziehungsweise aufsteigenden Ast der Schmelzlinie des neuen Bodenkörpers. Das Maximum liegt bei einer zwischenliegenden Zusammensetzung und entspricht ziemlich genau der $\mathrm{Zu}-$ sammensetzung einer Verbindung von 3 Molekülen Phenol und 2 Molekülen Diamin, d. i. $43 \cdot 3$ Gewichtsprozenten Diamin. Es liegt also eine Verbindung dieser Zusammensetzung vom homogenen maximalen Schmelzpunkt von $52 \cdot 6^{\circ}$ vor. Ihr Eutektikum mit Phenol liegt bei $24^{\circ}$ und $15 \%$ Diamin, das mit Diamin bei $41^{\circ}$ und $77 \%$ Diamin. Im System Phenol-o-Phenylendiamin liegen, wie die Daten der Tabelle 3 und ihre graphische Darstellung in Fig. 3 es zeigen, außer den Schmelzlinien der reinen Komponenten zwei neue Äste im Schmelzdiagramm auf, die also das Auftreten zweier Verbindungen im festen Zustande anzeigen. Die phenolreichere Verbindung zeigt ein äußerst stark abgeflachtes Maximum bei $29^{\circ}$, das nach seiner Lage einer Verbindung von 4 Molekülen Phenol und 1 Molekül Diamin entspricht, indem sich für eine solche Verbindung ein Gehait von $22.5 \%$ Diamin berechnet. Ihr Eutektikum mit Phenol liegt bei $28^{\circ}$ und $14 \%$ Diamin, das Eutektikum mit der zweiten nächst diaminreicheren Verbindung bei rund $29^{\circ}$ und $26 \%$. Diamin. 


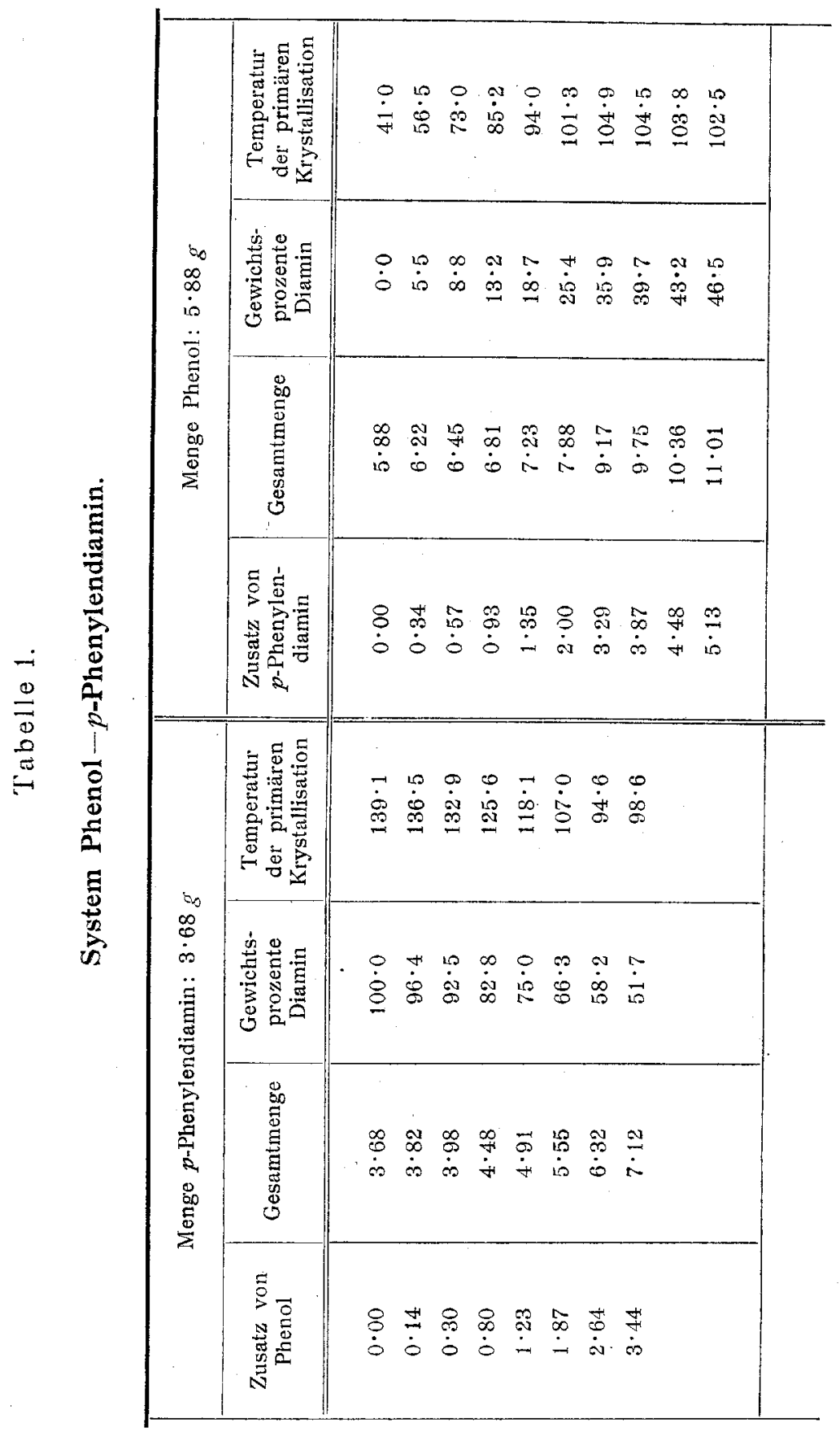




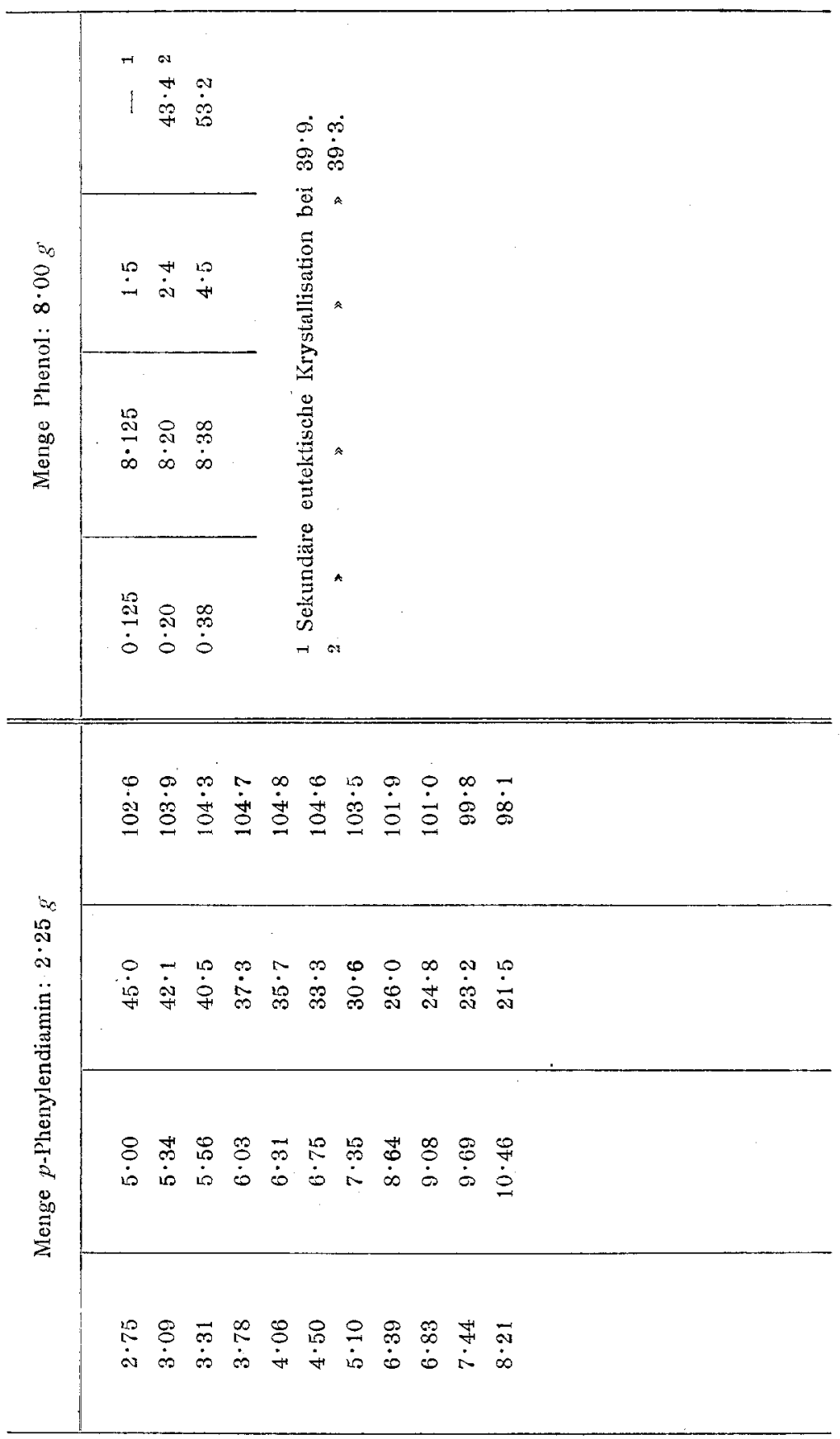




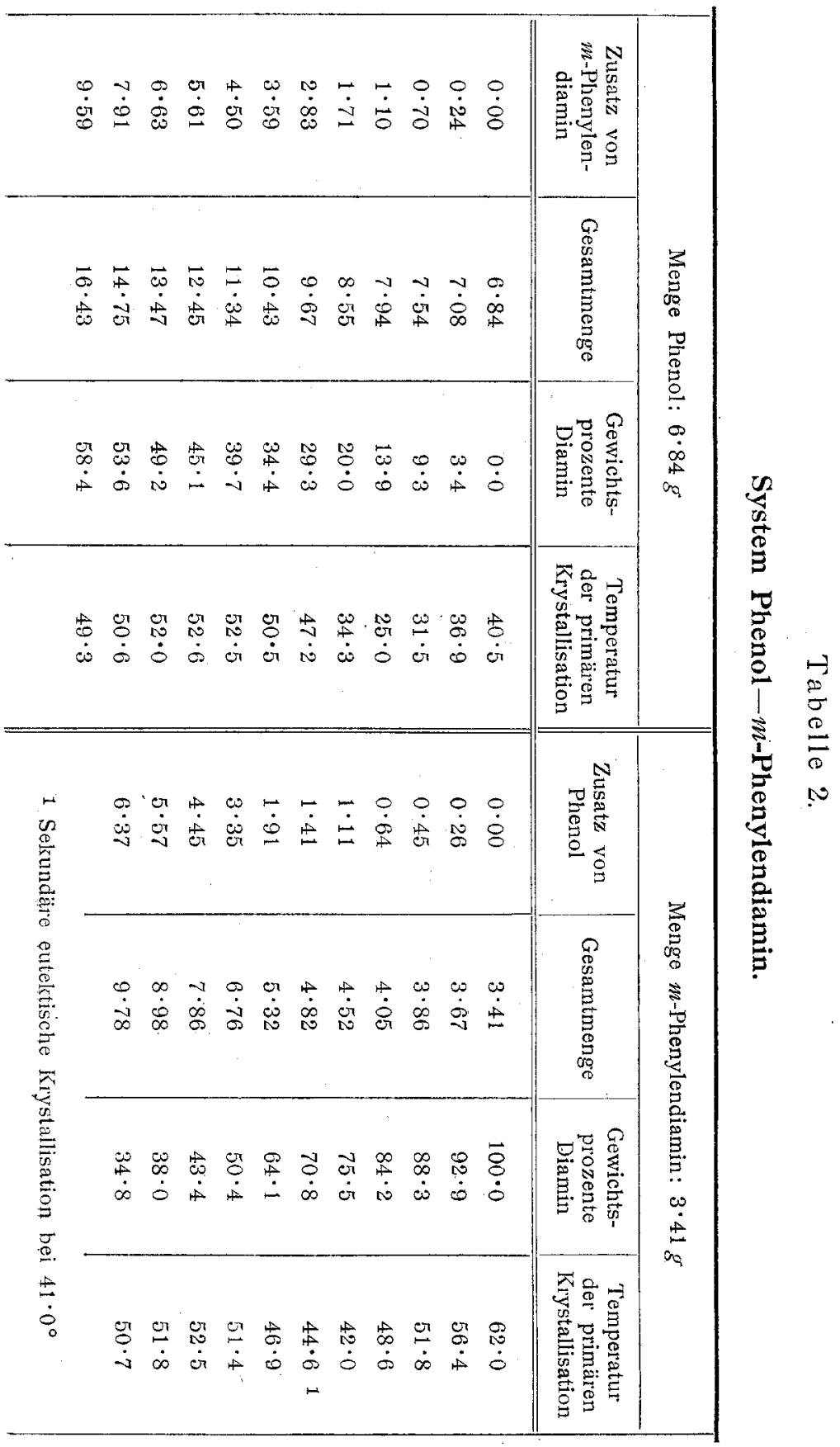


Was die zweite nächst diaminreichere Verbindung anlangt, so kann dieselbe keinesfalls der Zusammensetzung 2 Phenol.1 Diamin oder 3 Phenol.2 Diamin entsprechen, weil die diesen Verbindungen entsprechend zusammengesetzten Schmelzen bei $36 \cdot 7$, bezi ehungsweise $43 \cdot 3 \%$ Diamin noch im aufsteigenden Ast der Schmelzkurve liegen. Es kommt also als die nächst diaminreichere einfach zusammengesetzte Verbindung eine äquimolekulare in Betracht.

Diese einer Zusammensetzung der Schmelze von $53.5 \%$ Diamin entsprechende Verbindung schmilzt also nicht homogen, sondern scheidet beim Schmelzen primär Diamin ab. Der Umwandlungspunkt liegt bei $43.5^{\circ}$ und entspricht einem Gehalt der Schmelze an $48 \%$ Diamin. Mit steigendem Phenylendiaminzusatz beobachtet man stetig tiefere Temperaturen der Umwandlungserscheinung, was auf eine langsame Umwandlungsgeschwindigkeit bei gleichzeitiger mangelhafter Wärmeleitung zurückgeführt werden muß. Dies ist auch der Grund, weshalb wir hier wie in einigen anderen ähnlichen Fällen den üblichen Beweis für die Zusammensetzung solcher Verbindungen aus dem Maximum der Haltzeiten bei der Umwandlungstemperatur nicht durchführen konnten. Gleichwohl ist nach Obigem in diesem System neben der Verbindung von 4 Molekïllen Phenol auf 1 Molekül Diamin die Existenz einer äquimolekularen Verbindung als sicher anzusprechen. 


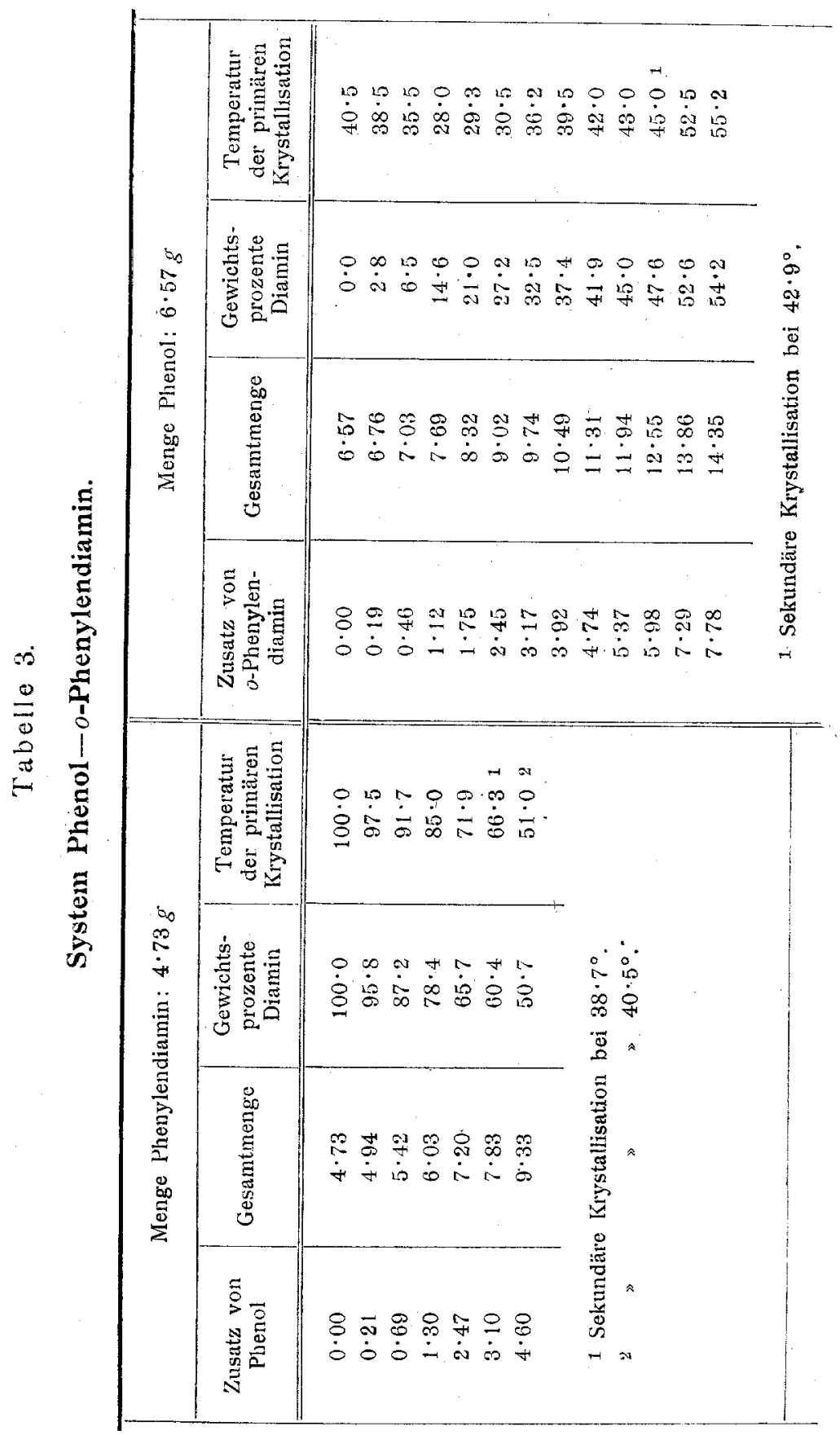




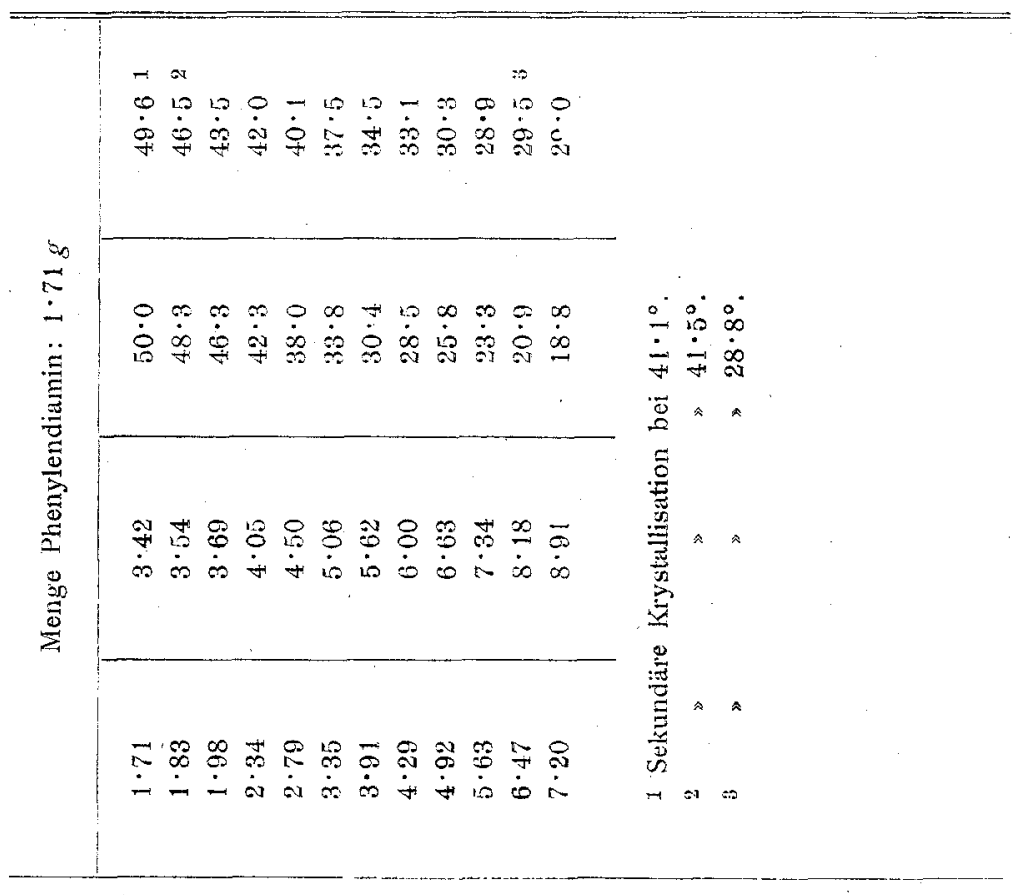


2. Systeme von $p$-Nitrophenol mit den drei isomeren Phenylendiaminen.

Aus den in Tabelle 4 wiedergegebenen und in Fig. 4 graphisch dargestellten Versuchsresultaten geht hervor, daß im System $p$-Nitrophenol-o-Phenylendiamin nur eine Verbindung vom homogenen maximalen Schmelzpunkt $87.9^{\circ}$ vorliegt der Zusammensetzung 2 Nitrophenol.1 o-Phenylendiamin, da bei der dieser Zusammensetzung entsprechenden Schmelze, d. i. bei 28 Gewichtsprozenten Diamin, die Schmelzlinie der Verbindung durch ein scharf ausgeprägtes Maximum läuft. Das Eutektikum der Verbindung mit $p$-Nitrophenol liegt bei $85.5^{\circ}$ und $19 \%$ Diamin, das Eutektikum mit $o$-Phenylendiamin bei $78^{\circ}$ und 54 bis $55 \%$ Phenylendiamin.

Ganz analoge Verhältnisse liegen, wie die in Fig. 5 dargestellten Versuchsergebnisse, wie sie in Tabelle 5 wiedergegeben sind, es zeigen, im System $p$-Nitrophenol - $m$-Phenylendiamin vor. Auch hier läuft die dem neuen Bodenkörper entsprechende Schmelzlinie durch ein Maximum bei 28 Gewichtsprozenten Diamin. Die Zusammensetzung der Verbindung vom homogenen maximalen Schmelzpunkt von 119:9 entspricht also auch hier dem Verhältnis von 2 Molekülen Nitrophenol und 1 Moleküu Diamin. Das Eutektikum dieser Verbindung mit Nitrophenol liegt bei $9 \%$ Diamin und $102^{\circ}$, das Eutektikum mit Diamin bei 80 Gewichtsprozenten Diamin und $52.4^{\circ}$. Der Grad der Abflachung des Maximums; also der Dissoziationsgrad der beiden Verbindungen beim Schmelzpunkt, ist bei beiden Verbindungen von Nitrophenol mit o-, beziehungsweise $m$-Diamin schätzungsweise gleich, obschon die Verbindung des o-Diamins um rund $30^{\circ}$ tiefer schmilzt. Bei gleichen Temperaturen dürfte also der Dissoziationsgrad der $o$-Verbindung größer, der der $m$-Verbindung kleiner sein.

Man kann also schließen, daß hier wieder die Orthoverbindung mit $p$-Nitrophenol unter einer geringeren Affinitätsäußerung $z \mathrm{u}$ einer Verbindung zusammentritt. Im System $p$-Nitrophenol $-p$-Phenylendiamin liegen, wie aus den in Tabelle 6 wiedergegebenen und in Fig. 6 graphisch dargestellten Versuchsergebnissen hervorgeht, zwei je durch ein 
Maximum gehende Äste der Schmelzlinie vor, die demnach das Auftreten zweier neuer Bodenkörper (Verbindungen) in diesem System anzeigen. Das eine Maximum bei $134 \cdot 2^{\circ}$ entspricht einer Zusammensetzung von rund $16.5 \%$ Diamin, das zweite bei $117 \cdot 5^{\circ}$ liegt bei einer Zusammensetzung zwischen 43 bis $44 \%$ Diamin.

Derartigen Zusammensetzungen der Schmelze entsprechen die Verbindungen von 4 Molekülen Nitrophenol und 1 Molekül Diamin, beziehungsweise 1 Molekül Nitrophenol und 1 Molekül Diamin mit Gehalten von $16 \cdot 3$, beziehungsweise $43 \cdot 7 \%$ Diamin. Es liegen also hier diese beiden Verbindungen mit den homogenen maximalen Schmelzpunkten von $134 \cdot 2$, beziehungsweise $117.5^{\circ}$ als Bodenkörper vor. Die Existenz einer Verbindung 2 Moleküle Nitrophenol.1 Molekül Diamin, die einer Zusammensetzung der Schmelze von $28 \%$ Diamin entspricht, ist hier ausgeschlossen, weil eine Schmelze dieser Zusammensetzung am absteigenden Ast der Schmelzlinie der nitrophenolreichen Verbindung liegt.

Beim Vergleich der Abflachung der beiden Maxima fällt auf, daß das der äquimolekularen Verbindung entsprechende Maximum eine weitaus stärkere Abflachung (größeren Dissoziationsgrad im Schmelzfluß) aufweist als das der nitrophenolreicheren Verbindung entsprechende, obwohl die erstere Verbindung um rund $17^{\circ}$ tiefer schmilzt. Wir dürfen daher annehmen, daß cet. par. die äquimolekulare Verbindung unter geringerer Affinitätswirkung sich bildet als die nitrophenolreichere Verbindung. Das Eutektikum der nitrophenolreicheren Verbindung mit $p$-Nitrophenol liegt bei rund 2 Gewichtsprozenten Diamin und $109.5^{\circ}$, das der äquimolekularen Verbindung mit der nitrophenolreichen Verbindung bei $42 \mathrm{Ge}$ wichtsprozenten Diamin und $1175^{\circ}$, das der äquimolekularen mit $p$-Phenylendiamin bei 62 Gewichtsprozenten und $107^{\circ}$. 


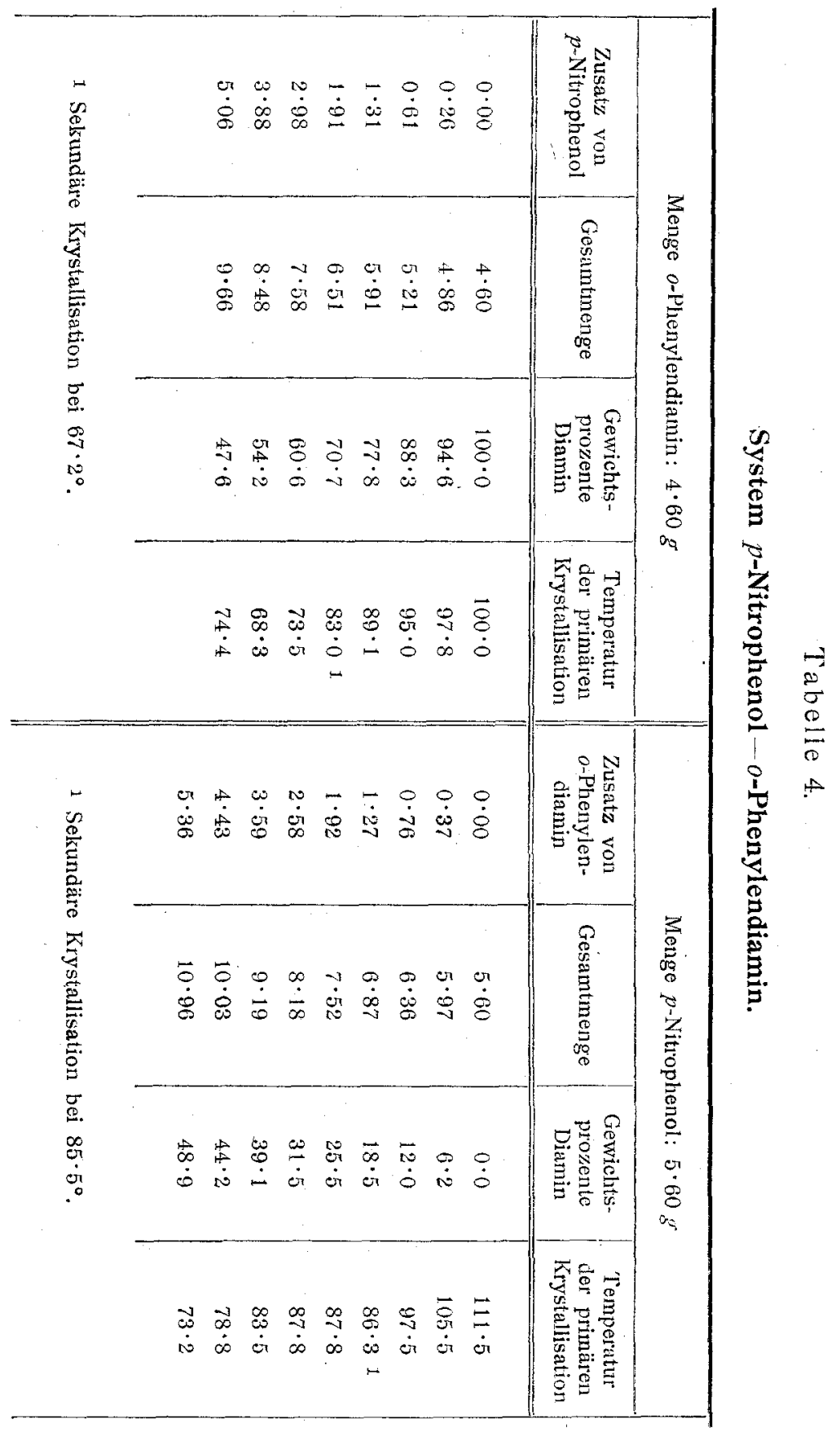


Lösungsgleichgewichte zwischen Phenol etc.

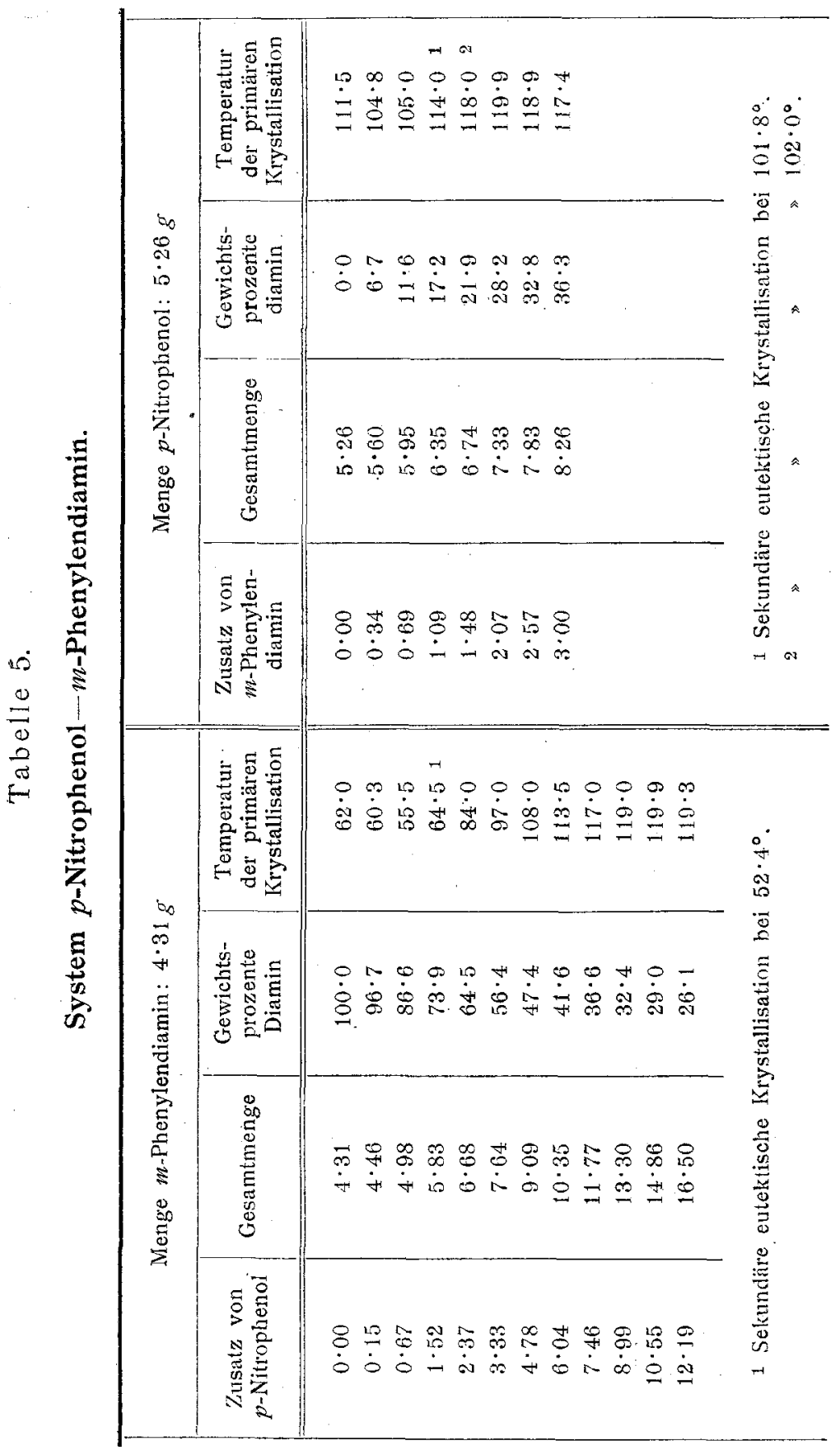




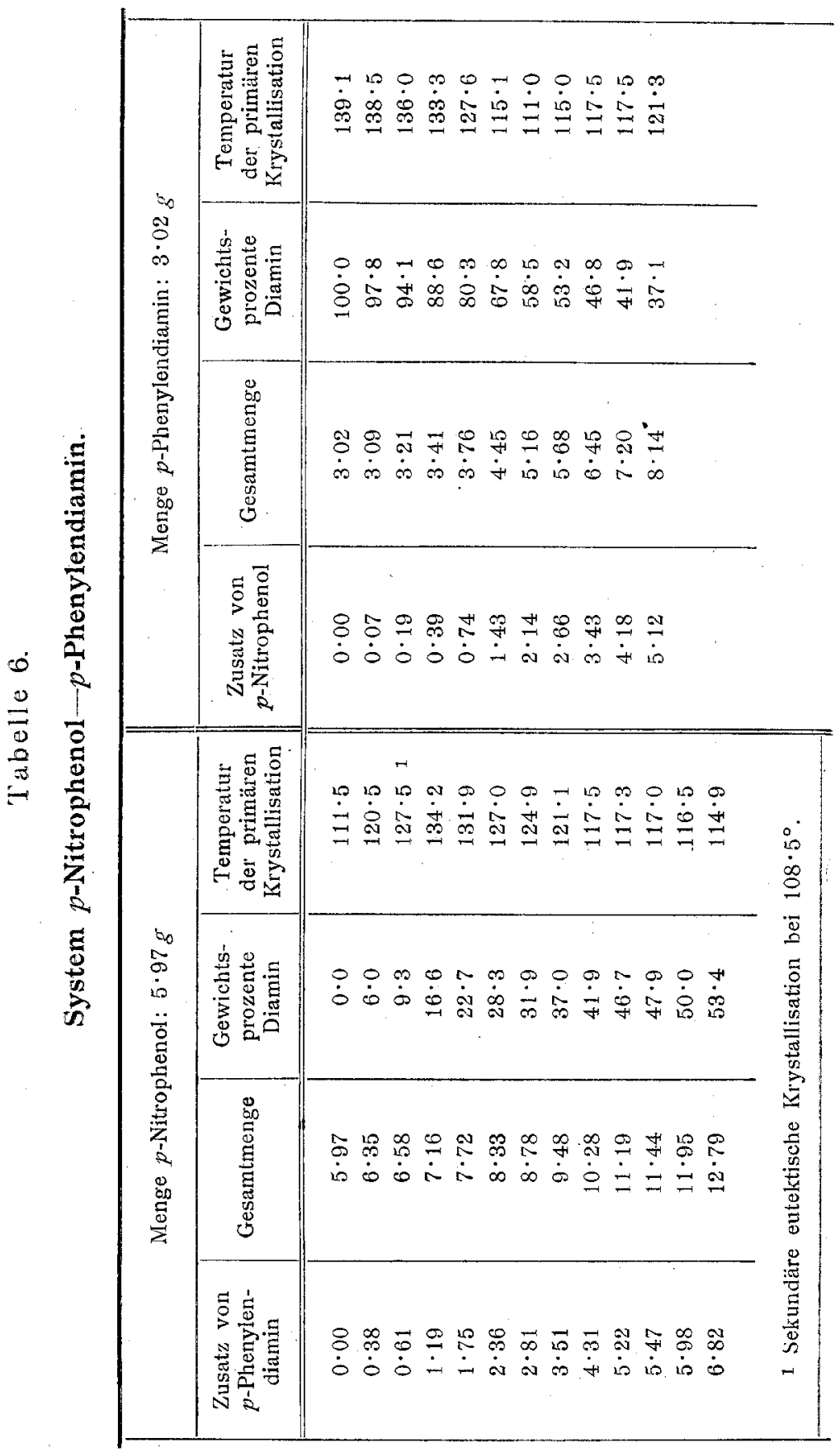




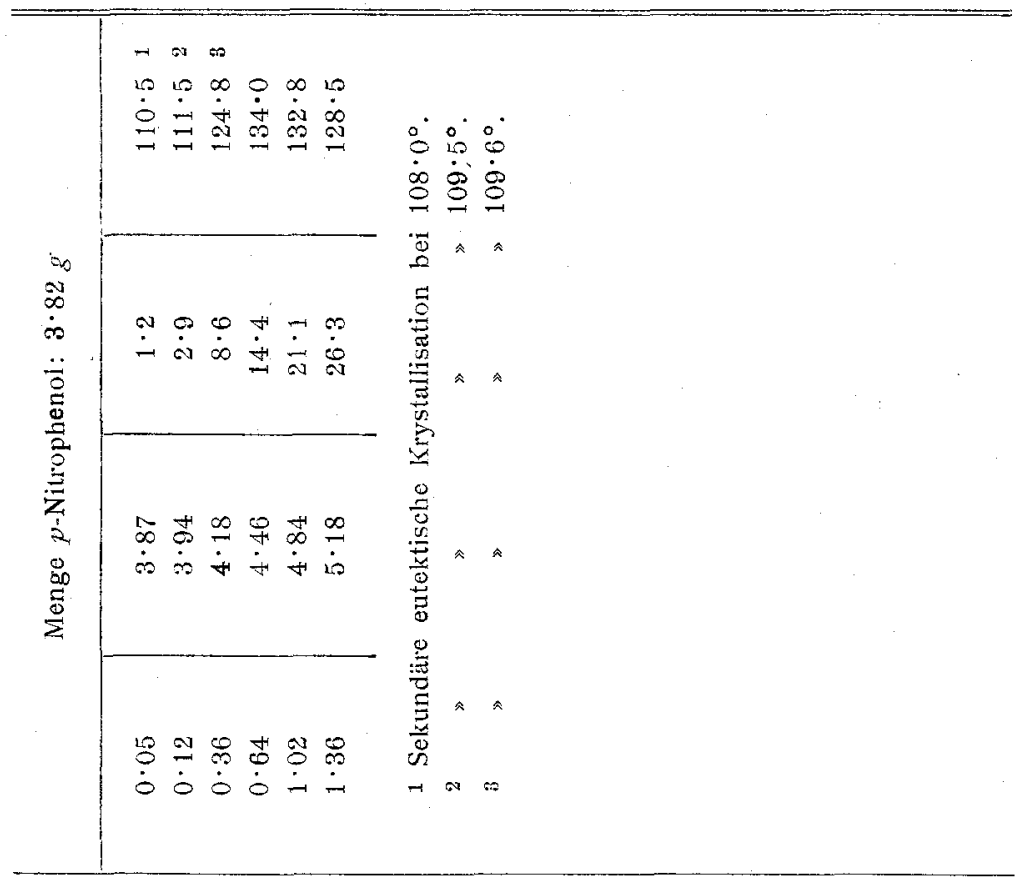


3. Die Systeme von o-Nitrophenol und den drei isomeren Phenylendiaminen.

Wie aus den in den Tabellen 7,8 und 9 wiedergegebenen und in den Figuren 7,8 und 9 graphisch dargestellten Versuchsresultaten hervorgeht, liegt in keinem dieser drei Systeme irgend ein Anzeichen für die Existenz von Verbindungen im festen Zustande vor, sondern nur einfache Eutektika, die folgenden Temperatur-Konzentrationspaaren entsprechen:

Im System $o$-Nitrophenol $-p$-Phenylendiamin bei $42 \cdot 5^{\circ}$ und $4 \cdot 5$ Gewichtsprozenten Diamin,

im System $o$-Nitrophenol- $m$-Phenylendiamin bei $33.5^{\circ}$ und 32 Gewichtsprozenten Diamin,

im System $o$-Nitrophenol-o-Phenylendiamin bei $38.8^{\circ}$ und 9 Gewichtsprozenten Diamin.

4. Die Systeme m-Nitrophenol und die drei isomeren Phenylendiamine.

Im System $m$-Nitrophenol- $m$-Phenylendiamin liegen außer den Schmelzlinien der reinen Komponenten, wie aus den in Fig. 10 graphisch dargestellten Versuchsergebnissen der Tabelle 10 hervorgeht, zwei durch je ein Maximum bei 28 Gewichtsprozenten Diamin und $74.5^{\circ}$, beziehungsweise 43.7 Gewichtsprozenten Diamin und $80.3^{\circ}$ gehende Schmelzlinien zweier Verbindungen vor, die nach der Lage der Maxima der Zusammensetzung 2 Moleküle Nitrophenol und 1 Molekül Diamin, beziehungsweise 1 Molekül Nitrophenol und 1 Molekül Diamin entsprechen. Letztere Verbindung zerfällt bei Überschuß von Phenylendiamin unterhalb $52^{\circ}$ in eine phenylendiaminreichere Verbindung, die sich unterhalb von $52^{\circ}$ und über Gehalten von $77 \cdot 5$ Gewichtsprozenten Diamin primär aus den Schmelzen abscheidet, bis zum Eutektikum dieser dritten phenylendiaminreicheren Verbindung bei $51^{\circ}$ und 84 Gewichtsprozenten Diamin. Die Existenz einer solchen Verbindung geht zweifelsohne aus der Tatsache hervor, daß eine Schmelze mit $72.9 \%$ Diamin einen sekundären Haltpunkt bei $52^{\circ}$ aufweist und eine Schmelze mit $82.1 \%$ Diamin bei $51.2^{\circ}$ primär $\mathrm{zu}$ erstarren beginnt und bei $51^{\circ}$ der eutektischen Temperatur dieser Verbindung mit Diamin vollends erstarrt ist. 
Lösungsgleichgewichte zwischen Phenol etc.

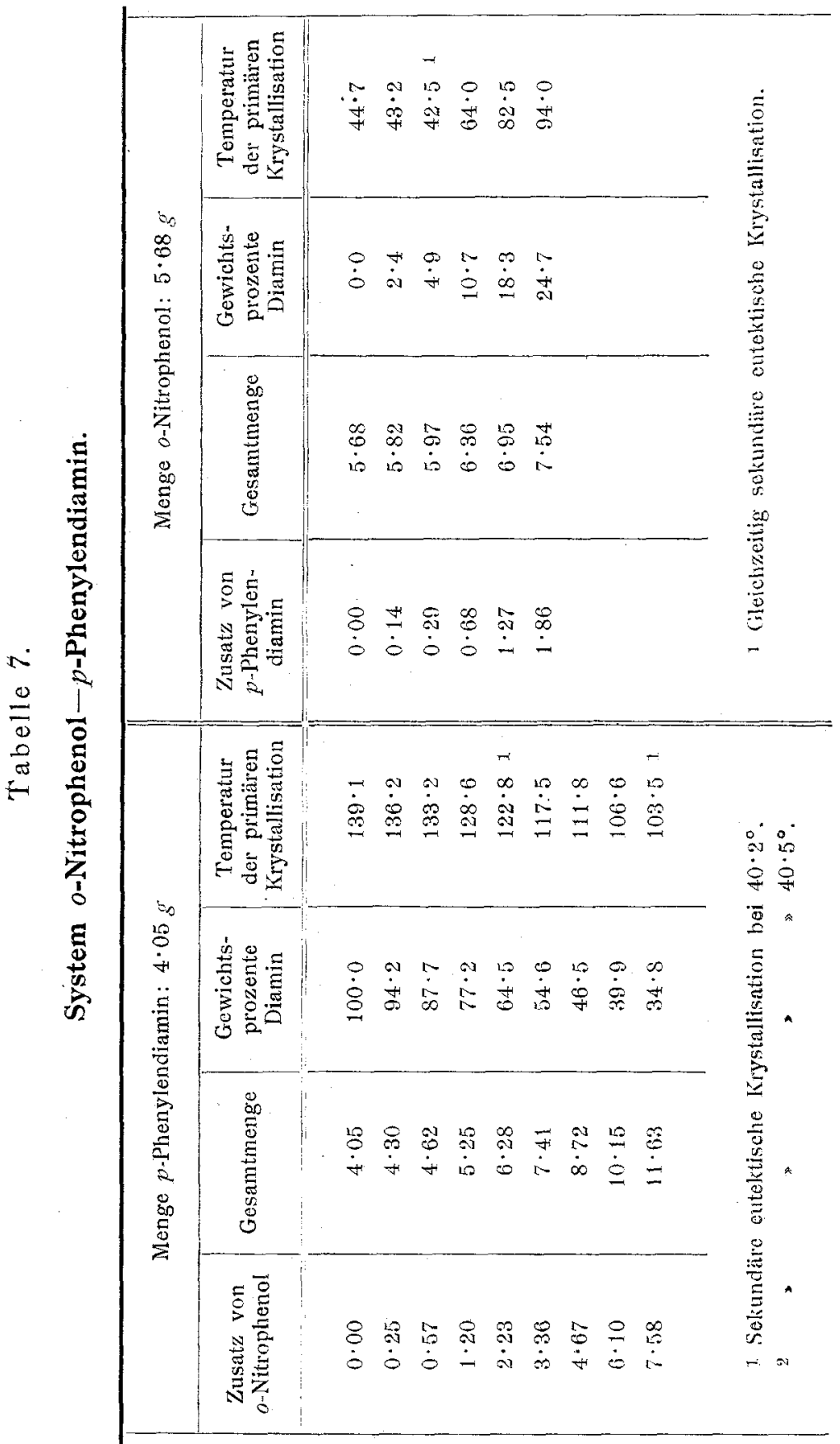




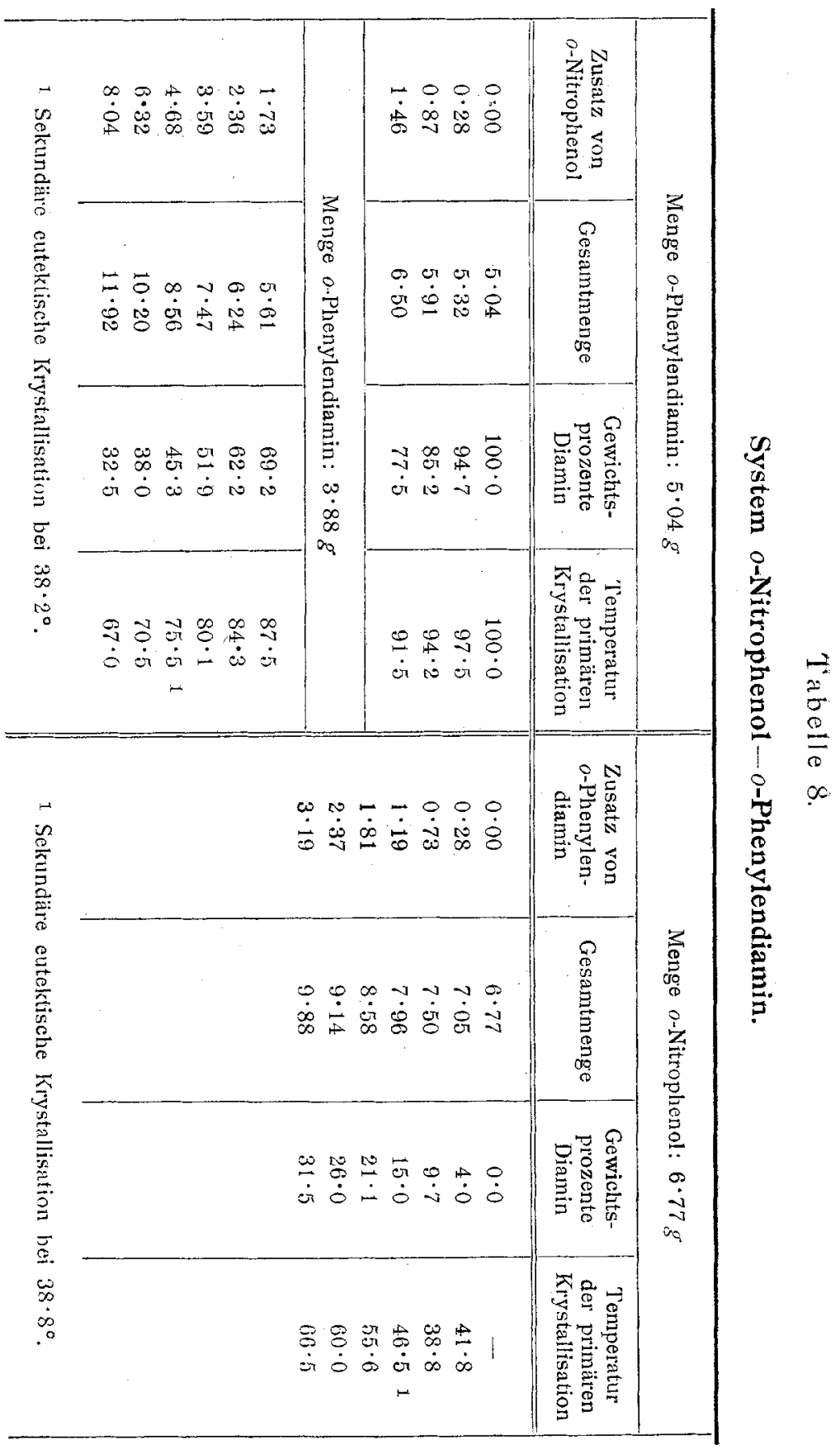


Lösungsgleichgewichte zwischen Phenol etc.

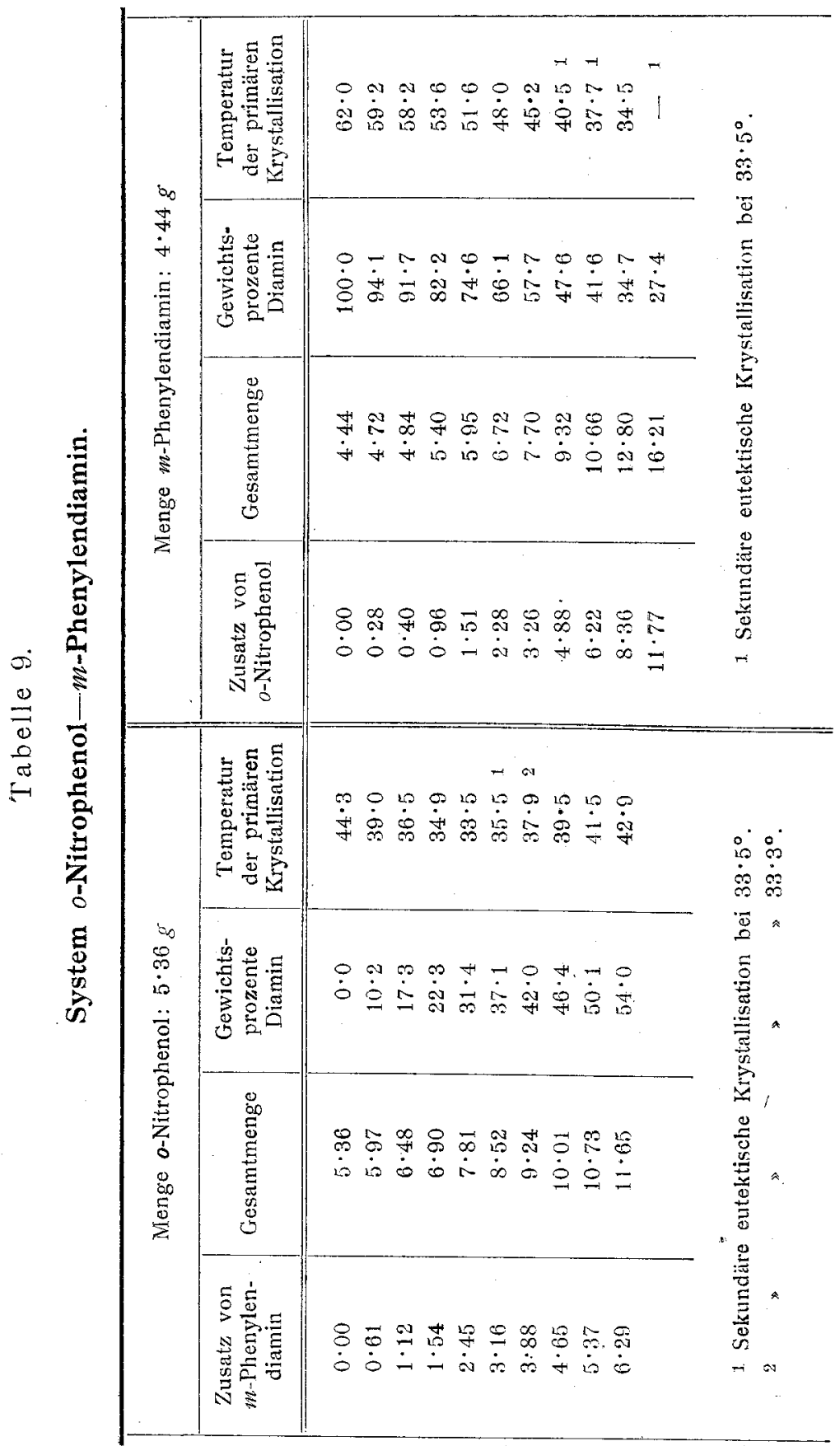




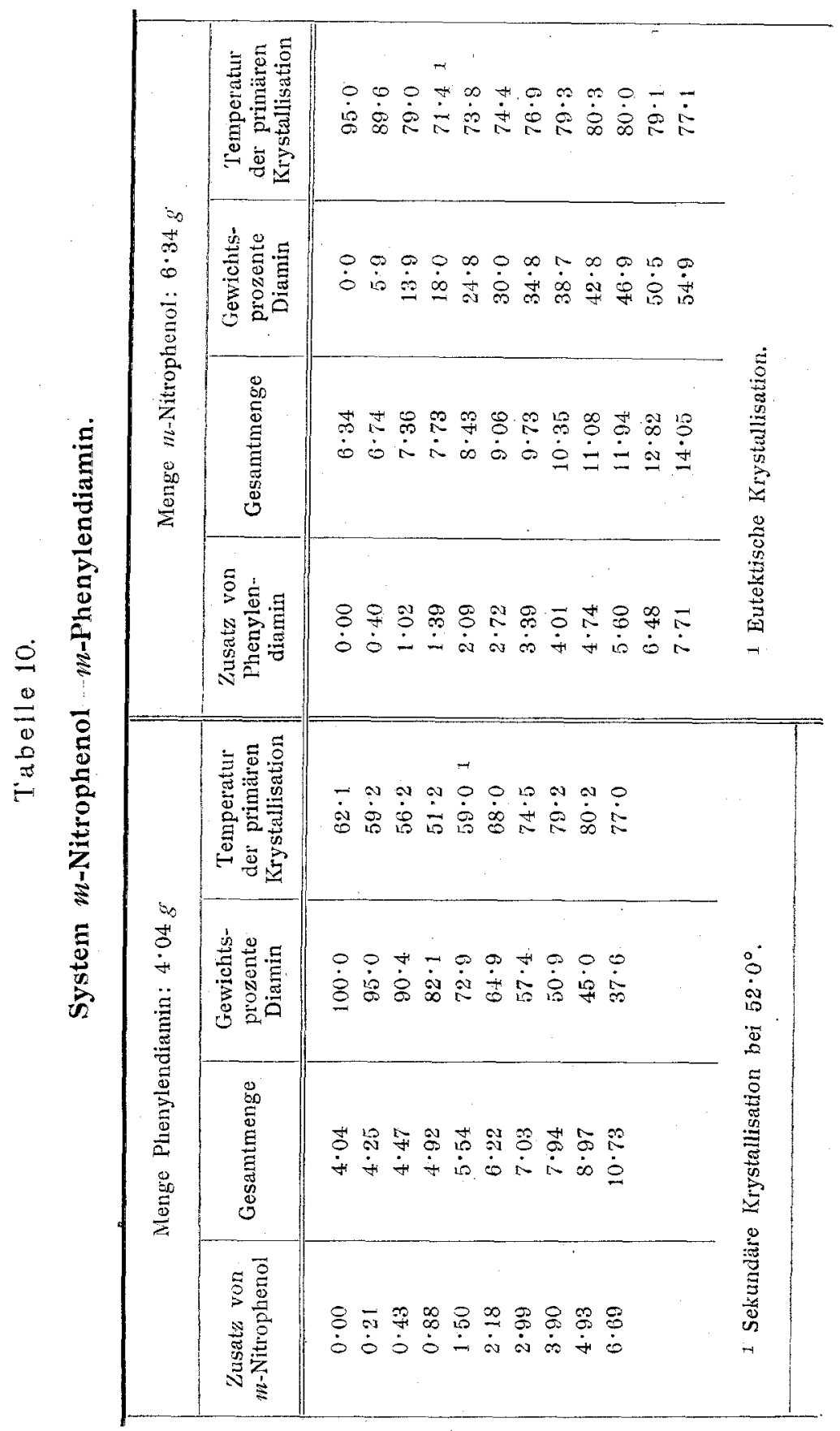




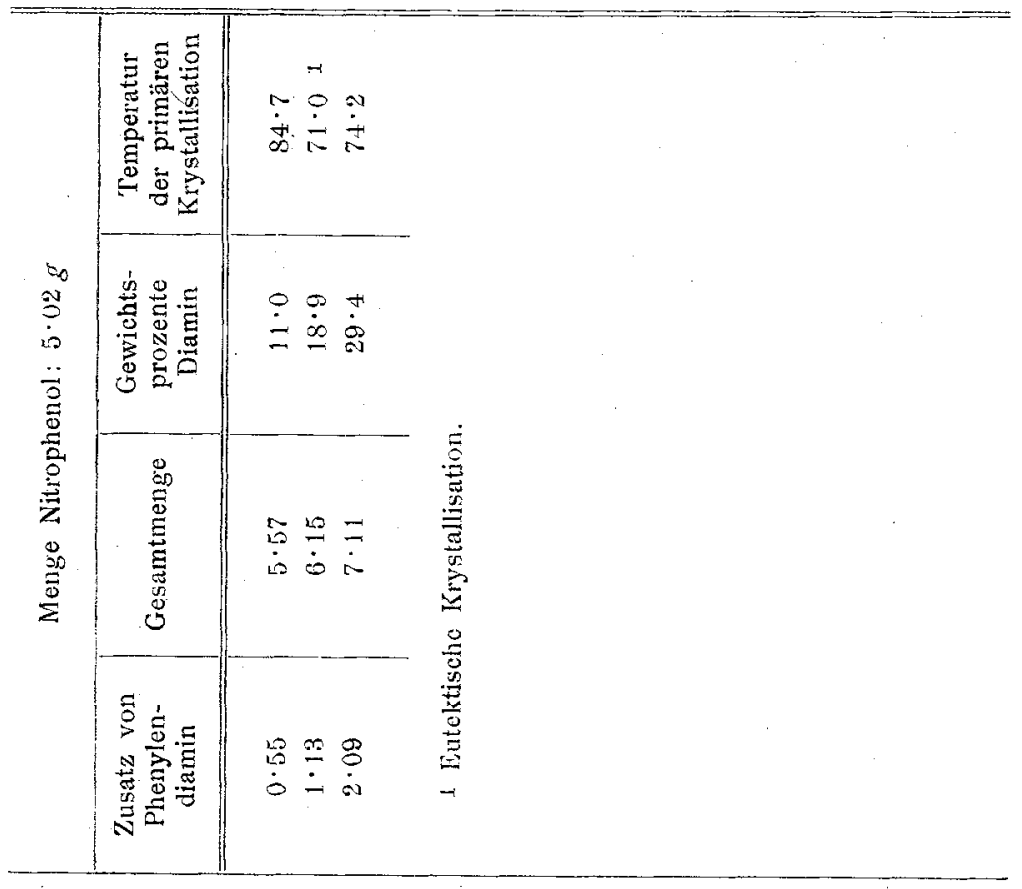


Würde im Konzentrationsbereich 77.5 bis 84 Gewichtsprozente Diamin keine neue Verbindung existieren, müßte ein Eutektikum zwischen der äquimolekularen Verbindung und Diamin vorliegen, das dem Schnittpunkt der in Fig. 10 gestrichelt gezeichneten Fortsetzung der Schmelzlinien dieser beiden Bodenkörper entsprechen müßte, der, wie aus Fig. 10 zu sehen ist, etwa bei $47.5^{\circ}$ und $81 \%$ Diamin liegen müßte. Dies stünde aber mit den oben erwähnten experimentellen Tatsachen im Widerspruch, so daß wir die Existenz einer dritten phenylendiaminreicheren Verbindung im festen $\mathrm{Zu}-$ stande als sicher erwiesen annehmen dürfen. Was ihre Zusammensetzung anlangt, so muß man erwägen, daß der ihr zukommende Ast der Schmelzlinie sehr wenig geneigt gegen die Konzentrationsachse verläuft, und zwar nach der diaminreicheren Seite abfällt. Wir dürfen also annehmen, daß der im Diagramm realisierbare Teil der Schmelzlinie dieser im Schmelzfluß weitgehend dissoziierten Verbindung ganz in der Nähe ihres Schmelzpunktes liegt.

Für die Zusammensetzung dieser Verbindung kommt daher das nächstliegende einfache molekulare Verhältnis der beiden Komponenten in Betracht, das links (also/nach der Seite der nitrophenolreichen Mischungen) vom Umwandlungspunkt bei $77 \cdot 5$ Gewichtsprozenten Diamin liegt. Einer Verbindung von 1 Molekül Nitrophenol und 4 Molekülen Diamin entspricht ein Gehalt von $77.4 \%$ Diamin. Wir dürfen also annehmen, daß eine Verbindung dieser Zusammensetzung als dritte im behandelten System als Bodenkörper vorliegt, deren Umwandlungspunkt mit ihrem Schmelzpunkt nahezu zusammenfällt. Das Eutektikum der nitrophenolreichsten Verbindung (2 Mole Nitrophenol.1 Diamin) mit reinem Nitrophenol liegt bei 18 Gewichtsprozenten Diamin und $71^{\circ}$, das Eutektikum dieser Verbindung mit der äquimolekularen bei $32 \%$ Diamin und rurd $74^{\circ}$.

Das System $m$-Nitrophenol-o-Phenylendiamin weist, wie aus den in Fig. 11 dargestellten Versuchsergebnissen der Tabelle 11 hervorgeht, eine durch ein Maximum bei $73.9^{\circ}$ bei einem Gehalt von 28 Gewichtsprozenten Diamin gehende, also der Verbindung 2 Moleküle $m$-Nitrophenol $-1 o$-Phenylen- 
diamin entsprechende Schmelzlinie auf. Das Eutektikum dieser Verbindung mit reinem $m$-Nitrophenol liegt bei $73.5^{\circ}$ und rund $18 \cdot 5$ Gewichtsprozenten Diamin. Die zweite in diesem System vorliegende Verbindung zeichnet sich durch ein infolge starker Dissoziation im Schmelzen nahezu horizontales Stück der Schmelzlinie aus, ein Kurvenverlauf, wie er das erste Mal von dem einen von uns für die äquimolekulare Verbindung Naphtalin-m-Dinitrobenzol ${ }^{1}$ und zum zweiten Male bloß für die äquimolekulare Verbindung $\beta$-Naphtylamin - 1,2, 4-Dinitrobenzol von dem einen von uns und G. Grasser² beobachtet wurde.

Dieses nahezu horizontale Kurvenstück, das von 45 bis 53 Gewichtsprozent Diamin reicht und einer Temperatur der primären Krystallisation von 63.2 bis $63.1^{\circ}$ entspricht, liegt am nächsten der Zusammensetzung einer äquimolekularen Verbindung beider Stoffe mit $43.7 \%$ Diamin. Zumal, wenn von einem Temperaturabfall dieses Kurvenstückes gesprochen werden kann, dieser nach der phenylendiaminreicheren Seite erfolgt, dürfen wir die äquimolekulare Verbindung als den längs des horizontalen Kurvenstückes vorliegenden Bodenkörper ansprechen. Dieser Verbindung entspricht also ein Umwandlungspunkt bei $45 \%$ Diamin und $63.2^{\circ}$ und ein Eutektikum mit $o$-Phenylendiamin bei rund $63 \cdot 1^{\circ}$ und $53 \%$ Diamin.

Ganz ähnliche Verhältnisse liegen vor im System $m$-Nitrophenol-p-Phenylendiamin. Außer dem durch ein deutliches Maximum der Schmelzpunktskurve bei 28 Gewichtsprozenten Diamin und $137 \cdot 8^{\circ}$, das also einer Verbindung von 2 Molekülen Nitrophenol und 1 Molekül Diamin entspricht, liegt, wie aus den in Fig. 12 dargestellten und in Tabelle 12 wiedergegebenen Versuchsresultaten $\mathrm{zu}$ ersehen ist, eine zweite phenylendiaminreichere Verbindung vor, die wiederum infolge weitgehender Dissoziation im Schmelzfluß und gleichzeitig geringem Existenzgebiet sich durch ein nahezu horizontales Stück der Schmelzlinie auszeichnet.

1 R. Kremann, Monatsh. f. Chemie, 25, 1246 (1904).

2 Monatsh. f. Chemie, 32, 734 (1916). 


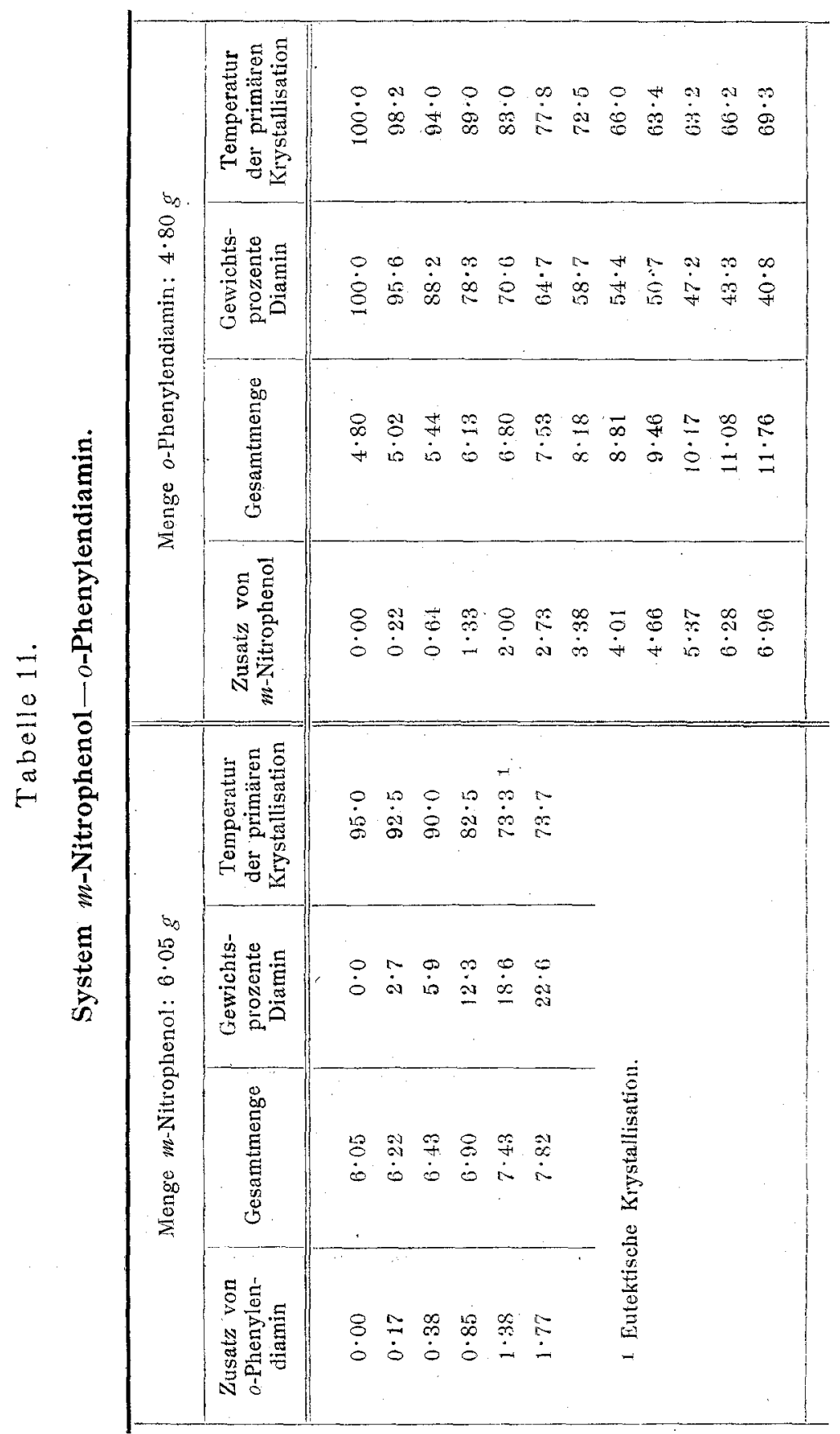




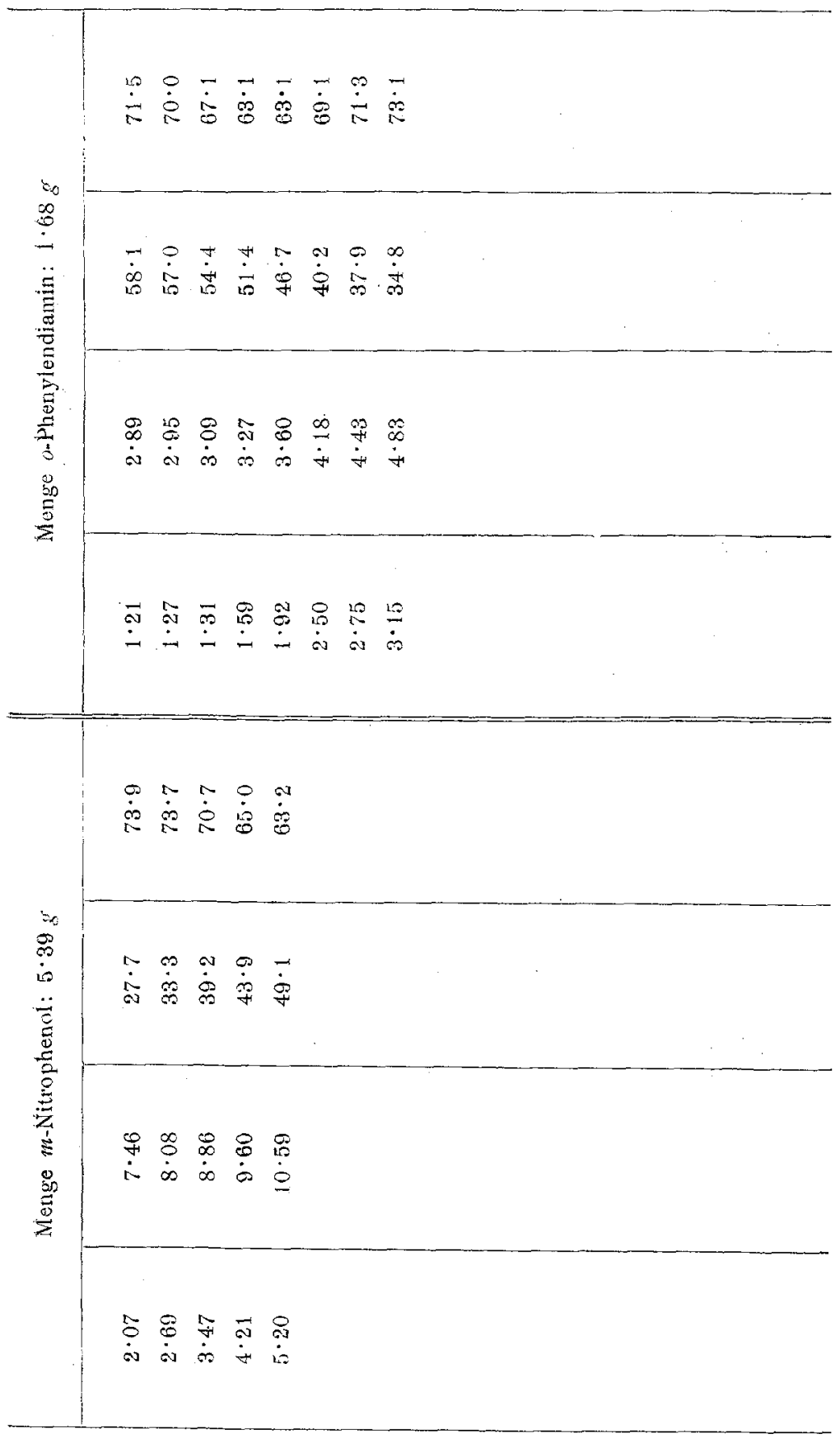




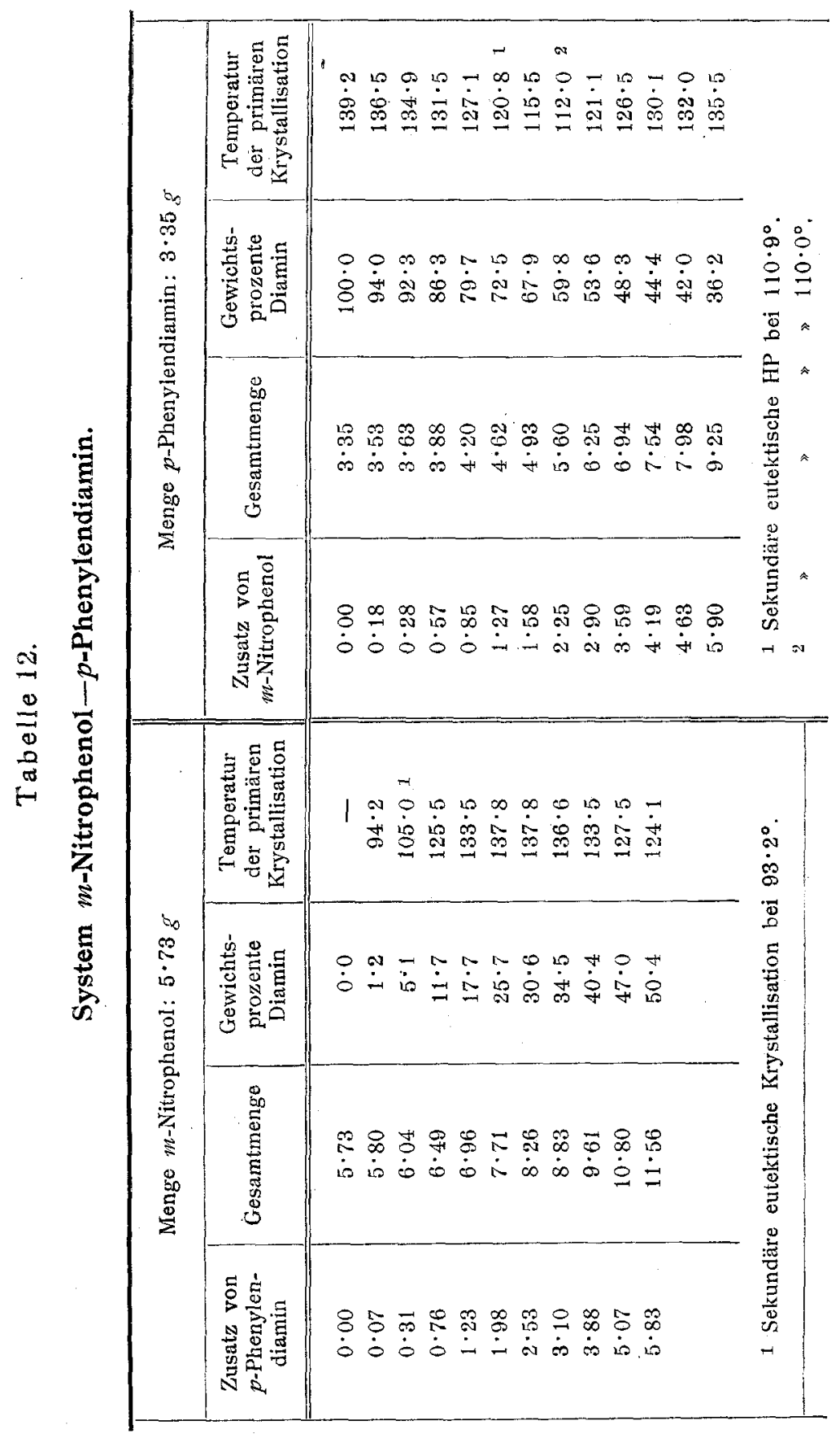




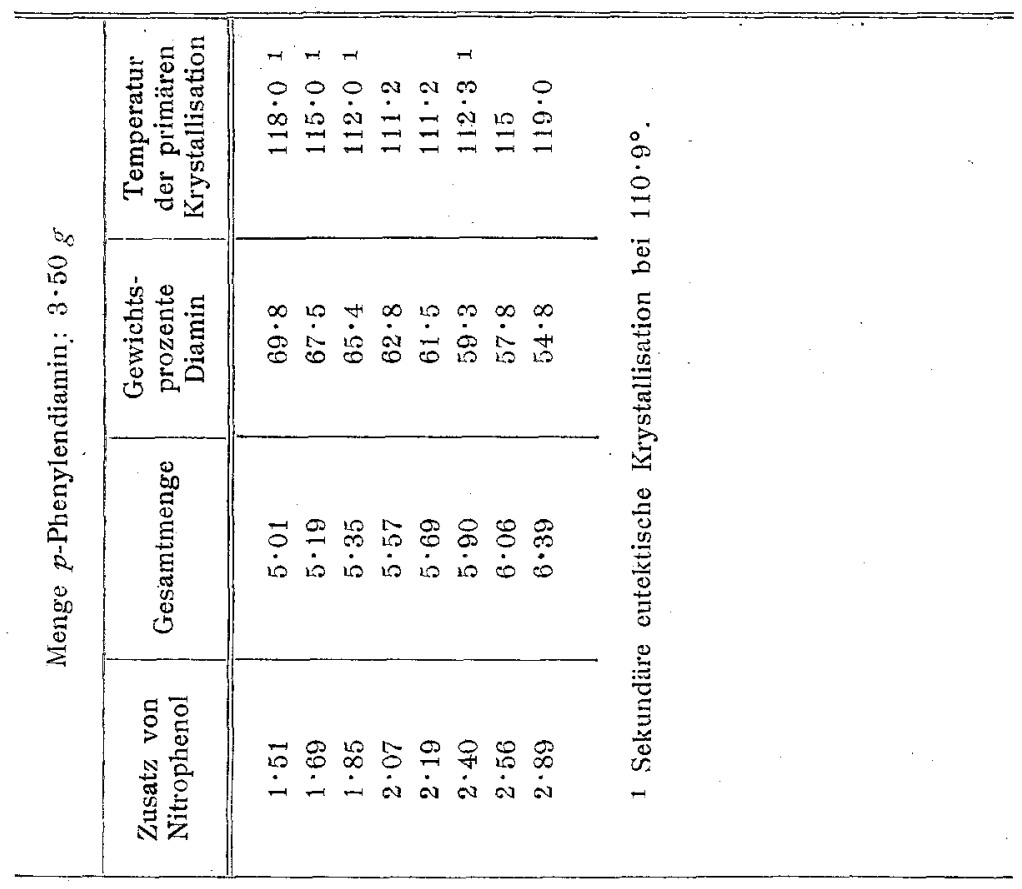


Da der Zusammensetzung einer Verbindung von 1 Moleküł Nitrophenol und 2 Molekülen Diamin gerade der eine Endpunkt dieses Kurvenstückes entspricht, die Zusammensetzung der übrigen einfacher zusammengesetzten Verbindungen aber mehr oder minder weit außerhalb dieser Kurve fällt, scheint es erlaubt, für die Zusammensetzung dieser zweiten phenylendiaminreicheren Verbindung die Zusammensetzung von 1 Molekül Nitrophenol und 2 Molekülen Diamin anzusprechen. Diese Verbindung scheidet sich also primär von ihrem Schmelzpunkt bei $112^{\circ}$ und 60.8 Gewichts prozenten Diamin bis zu. ihrem Eutektikum bei $111^{\circ}$ und $64.5 \%$ Diamin ab.

Vergleicht man in beiden letztgenannten Systemen von $m$-Nitrophenol mit $o$-, beziehungsweise $p$-Phenylendiamin den Beständigkeitsgrad der beiden in je einem System vorliegenden Verbindungen, so sieht man, daß jeweils die nitrophenolreichere Verbindung (je 2 Moleküle Nitrophenol und 1 Molekül Diamin) eine geringere Abflachung des Maximums der Schmelzlinie, also eine geringere Dissoziation im Schmelzfluß erleidet, als es der Fall ist bei den jeweiligen beiden phenylendiaminreicheren Verbindungen (1 Molekül $m$-Nitrophenol.1 Moleküil $o$-Phenylendiamin, beziehungsweise 1 Molekül $m$-Nitrophenol . 2 Moleküle $p$-Phenylendiamin), die sich nach Obigem durch weitgehende Dissoziation im Schmelzfluß auszeichnen (horizontales Kurvenstück).

Man kann also sagen, daß in den beiden Systemen die nitrophenolreicheren Verbindungen sich unter größerer Affinitätsäußerung bilden als die phenyl endiaminreicheren. 